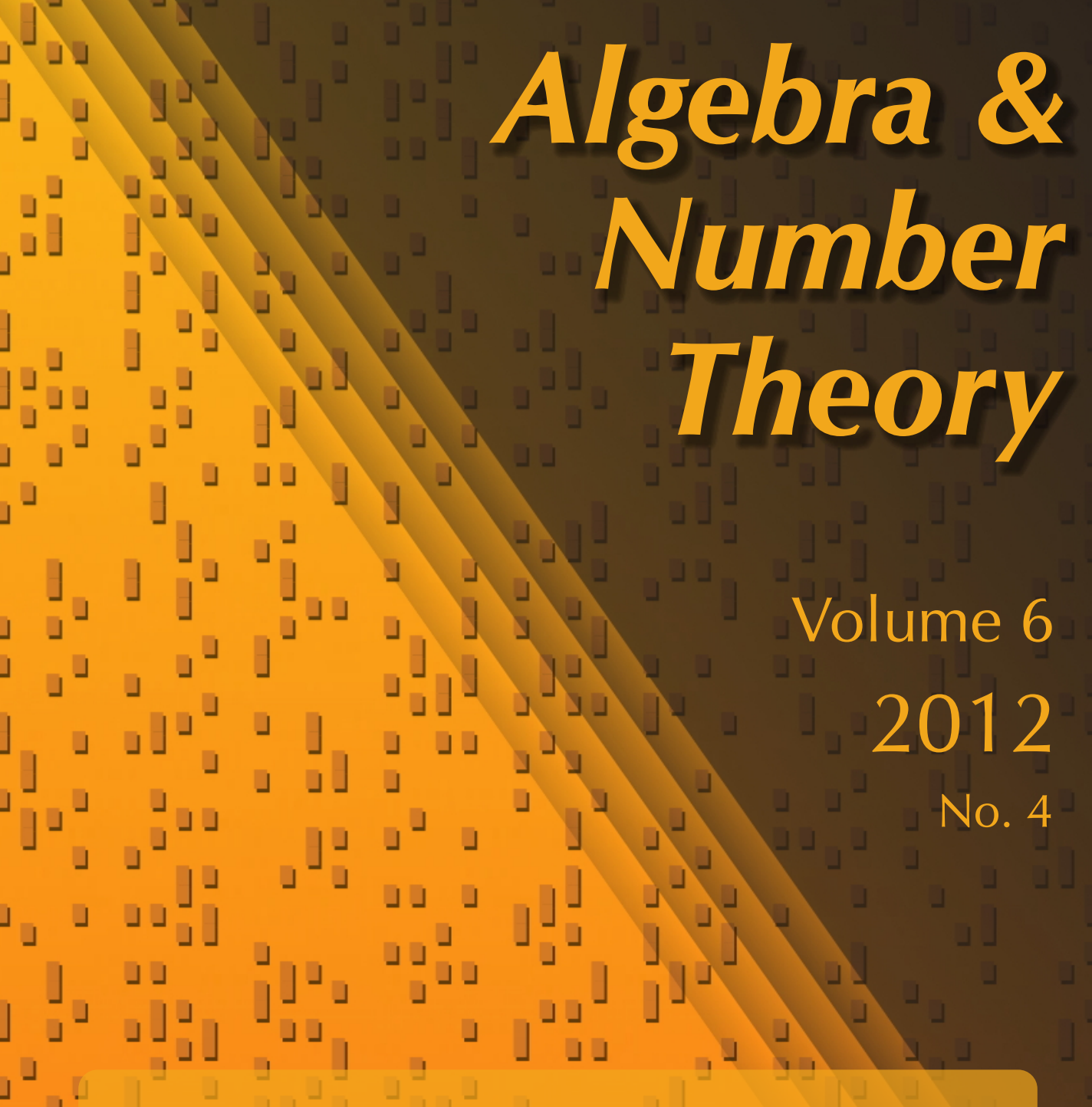

Basepoint-free theorems:

saturation, b-divisors, and canonical bundle formula

Osamu Fujino

」

\rfloor

\rfloor

.

\lrcorner

\lrcorner

\lrcorner \lrcorner$\rfloor$

\lrcorner$\lrcorner\lrcorner\lrcorner\lrcorner$

\lrcorner$\lrcorner\lrcorner\lrcorner\lrcorner\lrcorner\lrcorner$

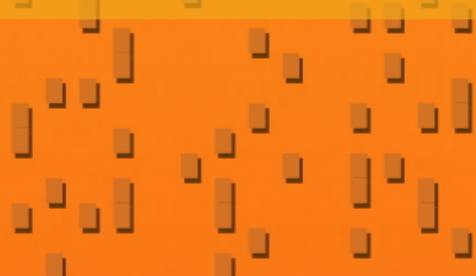




\title{
Basepoint-free theorems: saturation, b-divisors, and canonical bundle formula
}

\author{
Osamu Fujino
}

\begin{abstract}
We reformulate basepoint-free theorems using notions introduced by Shokurov, such as b-divisors and saturation of linear systems. Our formulation is flexible and has some important applications. One of the main purposes of this paper is to prove a generalization of the basepoint-free theorem in Fukuda's paper "On numerically effective log canonical divisors".
\end{abstract}

1. Introduction

2. Kawamata-Shokurov basepoint-free theorem revisited 800

3. b-divisors 802

4. Basepoint-free theorem; nef and abundant case 807

5. Basepoint-free theorem of Reid-Fukuda type 810

6. Variants of basepoint-free theorems due to Fukuda 813

7. Basepoint-free theorems for pseudo-klt pairs 817

Acknowledgement $\quad 821$

$\begin{array}{ll}\text { References } & 821\end{array}$

\section{Introduction}

In this paper, we reformulate basepoint-free theorems by using Shokurov's ideas [2003] of b-divisors and saturation of linear systems. Combining the refined Kawamata-Shokurov basepoint-free theorem (quoted here as Theorem 2.1) or its generalization (Theorem 6.1) with Ambro's formulation of Kodaira's canonical bundle formula, we obtain new basepoint-free theorems (Theorems 4.4 and 6.2), which are flexible and have some important applications (Theorem 7.11). One of the main purposes of this paper is to prove the following generalization of the basepoint-free theorem given in [Fukuda 2002, Proposition 3.3]:

Theorem 1.1. Let $(X, B)$ be an lc pair and let $\pi: X \rightarrow S$ be a proper morphism onto a variety $S$. Assume the following conditions:

MSC2010: primary 14C20; secondary 14N30, 14E30.

Keywords: basepoint-free theorem, canonical bundle formula, b-divisor, saturation. 
(A) H is a $\pi$-nef $\mathbb{Q}$-Cartier $\mathbb{Q}$-divisor on $X$.

(B) $H-\left(K_{X}+B\right)$ is $\pi$-nef and $\pi$-abundant.

(C) $\kappa\left(X_{\eta},\left(a H-\left(K_{X}+B\right)\right)_{\eta}\right) \geq 0$ and

$$
v\left(X_{\eta},\left(a H-\left(K_{X}+B\right)\right)_{\eta}\right)=v\left(X_{\eta},\left(H-\left(K_{X}+B\right)\right)_{\eta}\right)
$$

for some a $\in \mathbb{Q}$ with $a>1$, where $\eta$ is the generic point of $S$.

(D) There is a positive integer $c$ such that $\mathrm{cH}$ is Cartier and

$$
\mathrm{O}_{T}(c H):=\left.\mathrm{O}_{X}(c H)\right|_{T}
$$

is $\pi$-generated, where $T=\operatorname{Nklt}(X, B)$ is the non-klt locus of $(X, B)$.

Then $H$ is $\pi$-semiample.

This will be proved on page 816. As an application of Theorem 1.1, we have:

Theorem 1.2 [Fujino and Gongyo 2011, Theorem 4.12]. Let $\pi: X \rightarrow S$ be a projective morphism between projective varieties. Let $(X, B)$ be an lc pair such that $K_{X}+B$ is nef and $\log$ abundant over $S$. Then $K_{X}+B$ is $f$-semiample.

We also used Theorem 1.1 to prove the finite generation of the log canonical ring for log canonical 4-folds in [Fujino 2010]; see Remark 3.4 of that paper. As we explain elsewhere [Fujino 2007b, Remark 3.10.3; 2011d, 5.1], the proof of Theorem 4.3 of [Kawamata 1985] contains a gap. Because of that gap, Theorem 5.1 of [Kawamata 1985] was also not rigorously proved, and since Proposition 3.3 of [Fukuda 2002] depends on it, our proof of Theorem 1.1 is the first rigorous proof of this important result of Fukuda.

Another purpose of this paper is to show how to use Shokurov's ideas, such as b-divisors, saturation of linear systems, various kinds of adjunction, and so on, by reproving some known results in our formulation. Thus one can regard this paper as Chapter $8 \frac{1}{2}$ of the book [Corti et al. 2007]. It is also a complement of the paper [Fujino 2011d]. We do not use the powerful new method developed in [Ambro 2003; Fujino 2009a; 2009b; 2009c; 2011a; 2011b; 2011c]. For related topics and applications, see [Fujino 2010; Gongyo 2010, Section 6; Cacciola 2011; Fujino and Gongyo 2011].

Remark 1.3. Professor Yujiro Kawamata [2011a] has announced a correction to the error in the proof of [Kawamata 1985, Theorem 4.3]. The new proof seems to depend heavily on arguments in his preprints [2011b; 2010]. If we accept his correction, then Theorem 1.1 holds under the assumption that $(X, B)$ is dlt and $S$ is a point, by [Fukuda 2002, Proposition 3.3] (see Remark 6.7 (ii)). As stated in the introduction of [Kawamata 2011a], our arguments are simpler. We note that our approach is completely different from Kawamata's original one. Anyway, Theorem 1.1 plays a crucial role in our study of the log abundance conjecture for 
log canonical pairs; see [Fujino and Gongyo 2011, Section 4]. Therefore, this paper is very relevant for the minimal model program for log canonical pairs.

Let us explain the motivation for our formulation.

1.4. Motivation. Let $(X, B)$ be a projective klt pair and let $D$ be a nef Cartier divisor on $X$ such that $D-\left(K_{X}+B\right)$ is nef and big. Then the Kawamata-Shokurov basepoint-free theorem means that $|m D|$ is free for every $m \gg 0$. Let $f: Y \rightarrow X$ be a projective birational morphism from a normal projective variety $Y$ such that $K_{Y}+B_{Y}=f^{*}\left(K_{X}+B\right)$. We note that $f^{*} D$ is a nef Cartier divisor on $Y$ and that $f^{*} D-\left(K_{Y}+B_{Y}\right)$ is nef and big. It is obvious that $\left|m f^{*} D\right|$ is free for every $m \gg 0$ because $|m D|$ is free for every $m \gg 0$. In general, we cannot directly apply the Kawamata-Shokurov basepoint-free theorem to $f^{*} D$ and $\left(Y, B_{Y}\right)$. This is because $\left(Y, B_{Y}\right)$ is sub-klt but is not always klt. Note that a $\mathbb{Q}$-Cartier $\mathbb{Q}$-divisor $L$ on $X$ is nef, big, or semiample if and only if so is $f^{*} L$. However, the notion of klt is not stable under birational pull-backs. By adding a saturation condition, which is trivially satisfied for klt pairs, we can apply the Kawamata-Shokurov basepointfree theorem for sub-klt pairs (see Theorem 2.1). By this new formulation, the basepoint-free theorem becomes more flexible and has some important applications.

1.5. Background. A key result we need is [Ambro 2004, Theorem 0.2], which is a generalization of [Fujino 2003, Section 4: Pull-back of $L_{X / Y}^{s S}$. It originates from Kawamata's positivity theorem [1998] and Shokurov's idea of adjunction. For details, see [Ambro 2004, Introduction]. The formulation and calculation we borrow from [Ambro 2005b; 2007] grew out from Shokurov's saturation of linear systems $[2003,4.32]$.

1.6. Outline of the paper. In Section 2, we reformulate the Kawamata-Shokurov basepoint-free theorem for sub-klt pairs with a saturation condition. To state our theorem, we use the notion of b-divisors. It is very useful to discuss linear systems with some base conditions. In Section 3, we collect basic properties of b-divisors and prove some elementary properties. In Section 4, we discuss a slight generalization of the main theorem of [Kawamata 1985]. We need this generalization in Section 7. The main ingredient of our proof is Ambro's formulation of Kodaira's canonical bundle formula. By this formula and the refined Kawamata-Shokurov basepointfree theorem obtained in Section 2, we can quickly prove Kawamata's theorem in [Kawamata 1985] and its generalization without appealing to the notion of generalized normal crossing varieties. In Section 5, we treat the basepoint-free theorem of Reid-Fukuda type. In this case, the saturation condition behaves very well for inductive arguments. It helps us understand the saturation condition of linear systems. In Section 6, we prove some variants of basepoint-free theorems, mainly due to Fukuda [2002]. We reformulate them by using b-divisors and saturation 
conditions. Then we use Ambro's canonical bundle formula to reduce them to the easier case instead of proving them directly by the X-method. In Section 7, we generalize the Kawamata-Shokurov basepoint-free theorem and Kawamata's main theorem in [Kawamata 1985] for pseudo-klt pairs. Theorem 7.11, which is new, is the main theorem of this section. It will be useful for the study of lc centers (Theorem 7.13).

Notation. Let $B=\sum b_{i} B_{i}$ be a $\mathbb{Q}$-divisor on a normal variety $X$ such that $B_{i}$ is prime for every $i$ and that $B_{i} \neq B_{j}$ for $i \neq j$. We denote by

$$
\lceil B\rceil=\sum\left\lceil b_{i}\right\rceil B_{i}, \quad\lfloor B\rfloor=\sum\left\lfloor b_{i}\right\rfloor B_{i}, \quad \text { and }\{B\}=B-\lfloor B\rfloor
$$

the round-up, the round-down, and the fractional part of $B$. Note that we do not use $\mathbb{R}$-divisors in this paper. We make one general remark here. Since the freeness (or semiampleness) of a Cartier divisor $D$ on a variety $X$ depends only on the linear equivalence class of $D$, we can freely replace $D$ by a linearly equivalent divisor to prove the freeness (or semiampleness) of $D$.

We will work over an algebraically closed field $k$ of characteristic zero throughout this paper.

\section{Kawamata-Shokurov basepoint-free theorem revisited}

Kawamata and Shokurov claimed the following theorem for klt pairs, that is, they assumed that $B$ is effective, which implies that condition (2) is trivially satisfied. We think that our formulation is useful for some applications. Readers not familiar with the notion of b-divisors are referred to Section 3.

Theorem 2.1 (Basepoint-free theorem). Let $(X, B)$ be a sub-klt pair, let $\pi: X \rightarrow S$ be a proper surjective morphism onto a variety $S$ and let $D$ be a $\pi$-nef Cartier divisor on $X$. Assume the following conditions:

(1) $r D-\left(K_{X}+B\right)$ is nef and big over $S$ for some positive integer $r$.

(2) (Saturation condition.) There exists a positive integer $j_{0}$ such that

$$
\pi_{*} \mathrm{O}_{X}(\lceil\mathbf{A}(X, B)\rceil+j \bar{D}) \subseteq \pi_{*} \mathscr{O}_{X}(j D)
$$

for every integer $j \geq j_{0}$.

Then $m D$ is $\pi$-generated for every $m \gg 0$, that is, there exists a positive integer $m_{0}$ such that for every $m \geq m_{0}$ the natural homomorphism $\pi^{*} \pi_{*} \mathbb{O}_{X}(m D) \rightarrow \mathbb{O}_{X}(m D)$ is surjective. 
Proof. The usual proof of the basepoint-free theorem, that is, the X-method, works without any changes if we note Lemma 3.10. For the details, see, for example, [Kawamata et al. 1987, Section 3-1]. See also Remarks 3.14-3.17.

The assumptions in Theorem 2.1 are birational in nature. This point is indispensable in Section 4. We note that we can assume that $X$ is nonsingular and Supp $B$ is a simple normal crossing divisor because conditions (1) and (2) are invariant for birational pull-backs. So, it is easy to see that Theorem 2.1 is equivalent to the following theorem.

Theorem 2.2. Let $X$ be a nonsingular variety and let $B$ be $a \mathbb{Q}$-divisor on $X$ such that $\lfloor B\rfloor \leq 0$ and Supp $B$ is a simple normal crossing divisor. Let $\pi: X \rightarrow S$ be a projective morphism onto a variety $S$ and let $D$ be a $\pi$-nef Cartier divisor on $X$. Assume the following conditions:

(1) $r D-\left(K_{X}+B\right)$ is nef and big over $S$ for some positive integer $r$.

(2) (Saturation condition.) There exists a positive integer $j_{0}$ such that

$$
\pi_{*} \mathrm{O}_{X}(\lceil-B\rceil+j D) \simeq \pi_{*} \mathrm{O}_{X}(j D)
$$

for every integer $j \geq j_{0}$.

Then $m D$ is $\pi$-generated for every $m \gg 0$.

The following example says that the original Kawamata-Shokurov basepoint-free theorem does not necessarily hold for sub-klt pairs.

Example 2.3. Let $X=E$ be an elliptic curve. We take a Cartier divisor $H$ such that $\operatorname{deg} H=0$ and $l H \nsim 0$ for every $l \in \mathbb{Z} \backslash\{0\}$. In particular, $H$ is nef. We put $B=-P$, where $P$ is a closed point of $X$. Then $(X, B)$ is sub-klt and $H-\left(K_{X}+B\right)$ is ample. However, $H$ is not semiample. In this case, $H^{0}\left(X, O_{X}(\lceil\mathbf{A}(X, B)\rceil+j \bar{H})\right) \simeq$ $H^{0}\left(X, O_{X}(P+j H)\right) \simeq k$ for every $j$. However, $H^{0}\left(X, O_{X}(j H)\right)=0$ for all $j$. Therefore, the saturation condition in Theorem 2.1 does not hold.

We note that Kollár's effective basepoint-freeness holds under the same assumption as in Theorem 2.1.

Theorem 2.4 (Effective freeness). We use the same notation and assumption as in Theorem 2.1. Then there exists a positive integer $l$, which depends only on $\operatorname{dim} X$ and $\max \left\{r, j_{0}\right\}$, such that $l D$ is $\pi$-generated, that is, $\pi^{*} \pi_{*} \mathrm{O}_{X}(l D) \rightarrow \mathrm{O}_{X}(l D)$ is surjective.

Sketch of the proof. We need no new ideas. So, we just explain how to modify the arguments in [Kollár 1993, Section 2]. From now on, we use the notation in [Kollár 1993]. In that reference, $(X, \Delta)$ is assumed to be klt, that is, $(X, \Delta)$ is sub-klt and $\Delta$ is effective. The effectivity of $\Delta$ implies that $H^{\prime}$ is $f$-exceptional in [ibid., (2.1.4.3)]. We need this to prove $H^{0}\left(Y, O_{Y}\left(f^{*} N+H^{\prime}\right)\right)=H^{0}\left(X, \mathscr{O}_{X}(N)\right)$ in [ibid., (2.1.6)]. 
It is not difficult to see that $0 \leq H^{\prime} \leq\left\lceil\mathbf{A}(X, \Delta)_{Y}\right\rceil$ in our notation. Therefore, it is sufficient to assume the saturation condition Theorem 2.1(2) in the proof of Kollár's effective freeness (see [ibid., Section 2]). We make one more remark. Applying the argument in the first part of [ibid., 2.4] to $\mathcal{O}_{X}(j \bar{D}+\lceil\mathbf{A}(X, B)\rceil)$ on the generic fiber of $\pi: X \rightarrow S$ with the saturation condition (2) in Theorem 2.1, we obtain a positive integer $l_{0}$ that depends only on $\operatorname{dim} X$ and $\max \left\{r, j_{0}\right\}$ such that $\pi_{*} \mathbb{O}_{X}\left(l_{0} D\right) \neq 0$. As explained above, the arguments in Section 2 in [ibid.] work with only minor modifications in our setting. We leave the details as an exercise for the reader.

\section{3. b-divisors}

Let us recall the notion of singularities of pairs, referring the reader to [Fujino 2007b] for a more extended treatment.

Definition 3.1 (Singularities of pairs). Let $X$ be a normal variety and let $B$ be a $\mathbb{Q}$-divisor on $X$ such that $K_{X}+B$ is $\mathbb{Q}$-Cartier. Let $f: Y \rightarrow X$ be a resolution of singularities such that $\operatorname{Exc}(f) \cup f_{*}^{-1} B$ has a simple normal crossing support, where $\operatorname{Exc}(f)$ is the exceptional locus of $f$. We write

$$
K_{Y}=f^{*}\left(K_{X}+B\right)+\sum a_{i} A_{i} .
$$

We note that $a_{i}$ is called the discrepancy of $A_{i}$. Then the pair $(X, B)$ is sub-klt (resp. $s u b-l c$ ) if $a_{i}>-1$ (resp. $a_{i} \geq-1$ ) for every $i$. The pair $(X, B)$ is $k l t$ (resp. $l c$ ) if $(X, B)$ is sub-klt (resp. sub-lc) and $B$ is effective. (In some literature, sub-klt and sub-lc are sometimes called klt and lc.)

Let $(X, B)$ be an lc pair. If there exists a resolution $f: Y \rightarrow X$ such that $\operatorname{Exc}(f)$ and $\operatorname{Exc}(f) \cup f_{*}^{-1} B$ are simple normal crossing divisors on $Y$ and

$$
K_{Y}=f^{*}\left(K_{X}+B\right)+\sum a_{i} A_{i}
$$

with $a_{i}>-1$ for all $f$-exceptional $A_{i}$ 's, then $(X, B)$ is called $d l$ t.

Remark 3.2. Let $(X, B)$ be a klt (resp. lc) pair and let $f: Y \rightarrow X$ be a proper birational morphism of normal varieties. We put $K_{Y}+B_{Y}=f^{*}\left(K_{X}+B\right)$. Then $\left(Y, B_{Y}\right)$ is not necessarily klt (resp. lc) but it is sub-klt (resp. sub-lc).

Let us recall the definition of log canonical centers.

Definition 3.3 (Log canonical center). Let $(X, B)$ be a sub-lc pair. A subvariety $W \subset X$ is called a $\log$ canonical center or an lc center of $(X, B)$ if there is a resolution $f: Y \rightarrow X$ such that $\operatorname{Exc}(f) \cup \operatorname{Supp} f_{*}^{-1} B$ is a simple normal crossing divisor on $Y$ and a divisor $E$ with discrepancy -1 such that $f(E)=W$. A log canonical center $W \subset X$ of $(X, B)$ is called exceptional if there is a unique divisor $E_{W}$ on $Y$ with discrepancy -1 such that $f\left(E_{W}\right)=W$ and $f(E) \cap W=\varnothing$ for every other divisor $E \neq E_{W}$ on $Y$ with discrepancy -1; see [Kollár 2007, 8.1]. 
3.4. b-divisors. The notion of b-divisors, introduced by Shokurov, plays a central role in this paper, and we now recall its definition. For details, we refer to [Ambro 2005b, 1-B] and [Corti 2007, 2.3.2]. The reader can find various examples of b-divisors in [Iskovskikh 2003].

Definition 3.5 (b-divisor). Let $X$ be a normal variety and let $\operatorname{Div}(X)$ be the free abelian group generated by Weil divisors on $X$. A $b$-divisor on $X$ is an element

$$
\mathbf{D} \in \operatorname{Div}(X)=\operatorname{projlim}_{Y \rightarrow X} \operatorname{Div}(Y),
$$

where the projective limit is taken over all proper birational morphisms $f: Y \rightarrow X$ of normal varieties, under the push forward homomorphism $f_{*}: \operatorname{Div}(Y) \rightarrow \operatorname{Div}(X)$. A $\mathbb{Q}$-b-divisor on $X$ is an element of $\operatorname{Div}_{\mathbb{Q}}(X)=\operatorname{Div}(X) \otimes_{\mathbb{Z}} \mathbb{Q}$.

Definition 3.6 (Discrepancy $\mathbb{Q}$-b-divisor). Let $X$ be a normal variety and let $B$ be a $\mathbb{Q}$-divisor on $X$ such that $K_{X}+B$ is $\mathbb{Q}$-Cartier. Then the discrepancy $\mathbb{Q}$-b-divisor of the pair $(X, B)$ is the $\mathbb{Q}$-b-divisor $\mathbf{A}=\mathbf{A}(X, B)$ with the trace $\mathbf{A}_{Y}$ defined by the formula

$$
K_{Y}=f^{*}\left(K_{X}+B\right)+\mathbf{A}_{Y},
$$

where $f: Y \rightarrow X$ is a proper birational morphism of normal varieties.

Definition 3.7 (Cartier closure). Let $D$ be a $\mathbb{Q}$-Cartier $\mathbb{Q}$-divisor on a normal variety $X$. Then the $\mathbb{Q}$-b-divisor $\bar{D}$ denotes the Cartier closure of $D$, whose trace on $Y$ is $\bar{D}_{Y}=f^{*} D$, where $f: Y \rightarrow X$ is a proper birational morphism of normal varieties.

Definition 3.8. Let $\mathbf{D}$ be a $\mathbb{Q}$-b-divisor on $X$. The round up $\lceil\mathbf{D}\rceil \in \operatorname{Div}(X)$ is defined componentwise. The restriction of $\mathbf{D}$ to an open subset $U \subset X$ is a well-defined $\mathbb{Q}$-b-divisor on $U$, denoted by $\left.\mathbf{D}\right|_{U}$. Then $\mathscr{O}_{X}(\mathbf{D})$ is an $\mathscr{O}_{X}$-module whose sections on an open subset $U \subset X$ are given by

$$
H^{0}\left(U, \mathscr{O}_{X}(\mathbf{D})\right)=\left\{a \in k(X)^{\times} ;\left.(\overline{(a)}+\mathbf{D})\right|_{U} \geq 0\right\} \cup\{0\},
$$

where $k(X)$ is the function field of $X$. Note that $\mathscr{O}_{X}(\mathbf{D})$ is not necessarily coherent.

3.9. Basic properties. We recall the first basic property of discrepancy $\mathbb{Q}$-b-divisors. We will treat a generalization of Lemma 3.10 for sub-lc pairs below.

Lemma 3.10. Let $(X, B)$ be a sub-klt pair and let $D$ be a Cartier divisor on $X$. Let $f: Y \rightarrow X$ be a proper surjective morphism from a nonsingular variety $Y$. We write $K_{Y}=f^{*}\left(K_{X}+B\right)+\sum a_{i} A_{i}$. We assume that $\sum A_{i}$ is a simple normal crossing divisor. Then, for every integer $j$,

$$
\mathrm{O}_{X}(\lceil\mathbf{A}(X, B)\rceil+j \bar{D})=f_{*} \mathcal{O}_{Y}\left(\sum\left\lceil a_{i}\right\rceil A_{i}\right) \otimes \mathscr{O}_{X}(j D)
$$

Let $E$ be an effective divisor on $Y$ such that $E \leq \sum\left\lceil a_{i}\right\rceil A_{i}$. Then

$$
\pi_{*} f_{*} \mathrm{O}_{Y}\left(E+f^{*} j D\right) \simeq \pi_{*} \mathrm{O}_{X}(j D)
$$


if

$$
\pi_{*} O_{X}(\lceil\mathbf{A}(X, B)\rceil+j \bar{D}) \subseteq \pi_{*} O_{X}(j D),
$$

where $\pi: X \rightarrow S$ is a proper surjective morphism onto a variety $S$.

Proof. For the first equality, see [Corti 2007, Lemmas 2.3.14 and 2.3.15] or their generalizations: Lemmas 3.19 and 3.20 below. Since $E$ is effective,

$$
\pi_{*} \mathrm{O}_{X}(j D) \subseteq \pi_{*} f_{*} \mathrm{O}_{Y}\left(E+f^{*} j D\right) \simeq \pi_{*}\left(f_{*} \mathcal{O}_{Y}(E) \otimes \mathcal{O}_{X}(j D)\right) .
$$

By the assumption and $E \leq \sum\left\lceil a_{i}\right\rceil A_{i}$,

$$
\begin{aligned}
\pi_{*}\left(f_{*} \mathscr{O}_{Y}(E) \otimes \mathscr{O}_{X}(j D)\right) & \subseteq \pi_{*}\left(f_{*} \mathscr{O}_{Y}\left(\sum\left\lceil a_{i}\right\rceil A_{i}\right) \otimes \mathscr{O}_{X}(j D)\right) \\
& =\pi_{*} \mathscr{O}_{X}(\lceil\mathbf{A}(X, B)\rceil+j \bar{D}) \\
& \subseteq \pi_{*} \mathscr{O}_{X}(j D) .
\end{aligned}
$$

Therefore, we obtain $\pi_{*} f_{*} \mathrm{O}_{Y}\left(E+f^{*} j D\right) \simeq \pi_{*} \mathscr{O}_{X}(j D)$.

We will use Lemma 3.11 in Section 4. The vanishing theorem in Lemma 3.11 is nothing but the Kawamata-Viehweg-Nadel vanishing theorem.

Lemma 3.11. Let $X$ be a normal variety and let $B$ be a $\mathbb{Q}$-divisor on $X$ such that $K_{X}+B$ is $\mathbb{Q}$-Cartier. Let $f: Y \rightarrow X$ be a proper birational morphism from a normal variety $Y$. We put $K_{Y}+B_{Y}=f^{*}\left(K_{X}+B\right)$. Then

$$
f_{*} \mathrm{O}_{Y}\left(\left\lceil\mathbf{A}\left(Y, B_{Y}\right)\right\rceil\right)=\mathscr{O}_{X}(\lceil\mathbf{A}(X, B)\rceil)
$$

and

$$
R^{i} f_{*} \mathrm{O}_{Y}\left(\left\lceil\mathbf{A}\left(Y, B_{Y}\right)\right\rceil\right)=0
$$

for every $i>0$.

Proof. Let $g: Z \rightarrow Y$ be a resolution such that $\operatorname{Exc}(g) \cup g_{*}^{-1} B_{Y}$ has a simple normal crossing support. We put $K_{Z}+B_{Z}=g^{*}\left(K_{Y}+B_{Y}\right)$. Then $K_{Z}+B_{Z}=h^{*}\left(K_{X}+B\right)$, where $h=f \circ g: Z \rightarrow X$. By Lemma 3.10,

$$
\mathrm{O}_{Y}\left(\left\lceil\mathbf{A}\left(Y, B_{Y}\right)\right\rceil\right)=g_{*} \mathfrak{O}_{Z}\left(\left\lceil-B_{Z}\right\rceil\right)
$$

and

$$
\mathrm{O}_{X}(\lceil\mathbf{A}(X, B)\rceil)=h_{*} \mathrm{O}_{Z}\left(\left\lceil-B_{Z}\right\rceil\right) .
$$

Therefore, $f_{*} \mathcal{O}_{Y}\left(\left\lceil\mathbf{A}\left(Y, B_{Y}\right)\right\rceil\right)=\mathscr{O}_{X}(\lceil\mathbf{A}(X, B)\rceil)$. Since, $-B_{Z}=K_{Z}-h^{*}\left(K_{X}+B\right)$, we have

$$
\left\lceil-B_{Z}\right\rceil=K_{Z}+\left\{B_{Z}\right\}-h^{*}\left(K_{X}+B\right) .
$$

Therefore, $R^{i} g_{*} \mathrm{O}_{Z}\left(\left\lceil-B_{Z}\right\rceil\right)=0$ and $R^{i} h_{*} \mathrm{O}_{Z}\left(\left\lceil-B_{Z}\right\rceil\right)=0$ for every $i>0$ by the Kawamata-Viehweg vanishing theorem. Thus, $R^{i} f_{*} \mathcal{O}_{Y}\left(\left\lceil\mathbf{A}\left(Y, B_{Y}\right)\right\rceil\right)=0$ for every $i>0$ by Leray's spectral sequence. 
Remark 3.12. We use the same notation as in Remark 3.2. Let $(X, B)$ be a klt pair. Let $D$ be a Cartier divisor on $X$ and let $\pi: X \rightarrow S$ be a proper morphism onto a variety $S$. We put $p=\pi \circ f: Y \rightarrow S$. Then

$$
p_{*} O_{Y}\left(j f^{*} D\right) \simeq \pi_{*} O_{X}(j D) \simeq p_{*} O_{Y}\left(\left\lceil\mathbf{A}\left(Y, B_{Y}\right)\right\rceil+j \overline{f^{*} D}\right)
$$

for every integer $j$. This is because $f_{*} \mathscr{O}_{Y}\left(\left\lceil\mathbf{A}\left(Y, B_{Y}\right)\right\rceil\right)=\mathscr{O}_{X}(\lceil\mathbf{A}(X, B)\rceil) \simeq \mathcal{O}_{X}$ by Lemma 3.11.

Remark 3.13 (Multiplier ideal sheaf). Let $D$ be an effective $\mathbb{Q}$-divisor on a nonsingular variety $X$. Then $\mathrm{O}_{X}(\lceil\mathbf{A}(X, D)\rceil)$ is nothing but the multiplier ideal sheaf $\mathscr{f}(X, D) \subseteq \mathrm{O}_{X}$ of $D$ on $X$. See [Lazarsfeld 2004, Definition 9.2.1]. More generally, let $X$ be a normal variety and let $\Delta$ be a $\mathbb{Q}$-divisor on $X$ such that $K_{X}+\Delta$ is $\mathbb{Q}$-Cartier. Let $D$ be a $\mathbb{Q}$-Cartier $\mathbb{Q}$-divisor on $X$. Then $O_{X}(\lceil\mathbf{A}(X, \Delta+D)\rceil)=\mathscr{I}((X, \Delta) ; D)$, where the right hand side is the multiplier ideal sheaf defined (but not investigated) in [Lazarsfeld 2004, Definition 9.3.56]. In general, $\mathscr{O}_{X}(\lceil\mathbf{A}(X, \Delta+D)\rceil)$ is a fractional ideal of $k(X)$.

The next four remarks help us understand Theorem 2.1.

Remark 3.14 (Nonvanishing theorem). By Shokurov's nonvanishing theorem (see [Kawamata et al. 1987, Theorem 2-1-1]), we have $\pi_{*} \mathcal{O}_{X}(\lceil\mathbf{A}(X, B)\rceil+j \bar{D}) \neq 0$ for every $j \gg 0$. Thus $\pi_{*} \mathrm{O}_{X}(j D) \neq 0$ for every $j \gg 0$ by condition (2) in Theorem 2.1.

Remark 3.15. We know that $\lceil\mathbf{A}(X, B)\rceil \geq 0$ since $(X, B)$ is sub-klt. Therefore, $\pi_{*} O_{X}(j D) \subseteq \pi_{*} O_{X}(\lceil\mathbf{A}(X, B)\rceil+j \bar{D})$. This implies that

$$
\pi_{*} \mathrm{O}_{X}(j D) \simeq \pi_{*} \mathrm{O}_{X}(\lceil\mathbf{A}(X, B)\rceil+j \bar{D})
$$

for $j \geq j_{0}$, by condition (2) in Theorem 2.1.

Remark 3.16. If the pair $(X, B)$ is klt, then $\lceil\mathbf{A}(X, B)\rceil$ is effective and exceptional over $X$. In this case, it is obvious that $\pi_{*} O_{X}(j D)=\pi_{*} O_{X}(\lceil\mathbf{A}(X, B)\rceil+j \bar{D})$.

Remark 3.17. Condition (2) in Theorem 2.1 is a very elementary case of saturation of linear systems. See [Corti 2007, 2.3.3] and [Ambro 2005b, 1-D].

We next introduce the notion of non-klt $\mathbb{Q}$-b-divisor, which is trivial for sub-klt pairs. We will use this in Section 5.

Definition 3.18 (Non-klt $\mathbb{Q}$-b-divisor). Let $X$ be a normal variety and let $B$ be a $\mathbb{Q}$-divisor on $X$ such that $K_{X}+B$ is $\mathbb{Q}$-Cartier. Then the non-klt $\mathbb{Q}$-b-divisor of the pair $(X, B)$ is the $\mathbb{Q}$-b-divisor $\mathbf{N}=\mathbf{N}(X, B)$ with the trace $\mathbf{N}_{Y}=\sum_{a_{i} \leq-1} a_{i} A_{i}$ for

$$
K_{Y}=f^{*}\left(K_{X}+B\right)+\sum a_{i} A_{i},
$$

where $f: Y \rightarrow X$ is a proper birational morphism of normal varieties. It is easy to see that $\mathbf{N}(X, B)$ is a well-defined $\mathbb{Q}$-b-divisor. We put $\mathbf{A}^{*}(X, B)=\mathbf{A}(X, B)-\mathbf{N}(X, B)$. 
Of course, $\mathbf{A}^{*}(X, B)$ is a well-defined $\mathbb{Q}$-b-divisor and $\left\lceil\mathbf{A}^{*}(X, B)\right\rceil \geq 0$. If $(X, B)$ is sub-klt, then $\mathbf{N}(X, B)=0$ and $\mathbf{A}(X, B)=\mathbf{A}^{*}(X, B)$.

The next lemma is a generalization of Lemma 3.10.

Lemma 3.19. Let $(X, B)$ be a sub-lc pair and let $f: Y \rightarrow X$ be a resolution such that $\operatorname{Exc}(f) \cup \operatorname{Supp} f_{*}^{-1} B$ is a simple normal crossing divisor on $Y$. We write $K_{Y}=f^{*}\left(K_{X}+B\right)+\sum a_{i} A_{i}$. Then

$$
\mathcal{O}_{X}\left(\left\lceil\mathbf{A}^{*}(X, B)\right\rceil\right)=f_{*} \mathscr{O}_{Y}\left(\sum_{a_{i} \neq-1}\left\lceil a_{i}\right\rceil A_{i}\right) .
$$

In particular, $\mathrm{O}_{X}\left(\left\lceil\mathbf{A}^{*}(X, B)\right\rceil\right)$ is a coherent $\mathrm{O}_{X}$-module. If $(X, B)$ is $l c$, then $\mathrm{O}_{X}\left(\left\lceil\mathbf{A}^{*}(X, B)\right\rceil\right) \simeq \mathrm{O}_{X}$.

Let $D$ be a Cartier divisor on $X$ and let $E$ be an effective divisor on $Y$ such that $E \leq \sum_{a_{i} \neq-1}\left\lceil a_{i}\right\rceil A_{i}$. Then

$$
\pi_{*} f_{*} O_{Y}\left(E+f^{*} j D\right) \simeq \pi_{*} O_{X}(j D)
$$

if

$$
\pi_{*} \mathrm{O}_{X}\left(\left\lceil\mathbf{A}^{*}(X, B)\right\rceil+j \bar{D}\right) \subseteq \pi_{*} \mathrm{O}_{X}(j D),
$$

where $\pi: X \rightarrow S$ is a proper morphism onto a variety $S$.

Proof. By definition, $\mathbf{A}^{*}(X, B)_{Y}=\sum_{a_{i} \neq-1} a_{i} A_{i}$. If $g: Y^{\prime} \rightarrow Y$ is a proper birational morphism from a normal variety $Y^{\prime}$, then

$$
\left\lceil\mathbf{A}^{*}(X, B)_{Y^{\prime}}\right\rceil=g^{*}\left\lceil\mathbf{A}^{*}(X, B)_{Y}\right\rceil+F,
$$

where $F$ is a $g$-exceptional effective divisor, by Lemma 3.20 below. This implies $f_{*} O_{Y}\left(\left\lceil\mathbf{A}^{*}(X, B)_{Y}\right\rceil\right)=f_{*}^{\prime} O_{Y^{\prime}}\left(\left\lceil\mathbf{A}^{*}(X, B)_{Y^{\prime}}\right\rceil\right)$, where $f^{\prime}=f \circ g$, from which it follows that $\mathrm{O}_{X}\left(\left\lceil\mathbf{A}^{*}(X, B)\right\rceil\right)=f_{*} \mathcal{O}_{Y}\left(\sum_{a_{i} \neq-1}\left\lceil a_{i}\right\rceil A_{i}\right)$ is a coherent $\mathrm{O}_{X}$-module. The last statement is easy to check.

Lemma 3.20. Let $(X, B)$ be a sub-lc pair and let $f: Y \rightarrow X$ be a resolution as in Lemma 3.19. We consider the $\mathbb{Q}$-b-divisor $\mathbf{A}^{*}=\mathbf{A}^{*}(X, B)=\mathbf{A}(X, B)-\mathbf{N}(X, B)$. If $Y^{\prime}$ is a normal variety and $g: Y^{\prime} \rightarrow Y$ is a proper birational morphism, then

$$
\left\lceil\mathbf{A}_{Y^{\prime}}^{*}\right\rceil=g^{*}\left\lceil\mathbf{A}_{Y}^{*}\right\rceil+F,
$$

where $F$ is a g-exceptional effective divisor.

Proof. By definition, we have $K_{Y}=f^{*}\left(K_{X}+B\right)+\mathbf{A}_{Y}$. Therefore we may write

$$
\begin{aligned}
K_{Y^{\prime}} & =g^{*} f^{*}\left(K_{X}+B\right)+\mathbf{A}_{Y^{\prime}}=g^{*}\left(K_{Y}-\mathbf{A}_{Y}\right)+\mathbf{A}_{Y^{\prime}} \\
& =g^{*}\left(K_{Y}+\left\{-\mathbf{A}_{Y}^{*}\right\}-\mathbf{N}_{Y}+\left\lfloor-\mathbf{A}_{Y}^{*}\right\rfloor\right)+\mathbf{A}_{Y^{\prime}} \\
& =g^{*}\left(K_{Y}+\left\{-\mathbf{A}_{Y}^{*}\right\}-\mathbf{N}_{Y}\right)+\mathbf{A}_{Y^{\prime}}-g^{*}\left\lceil\mathbf{A}_{Y}^{*}\right\rceil .
\end{aligned}
$$


We note that $\left(Y,\left\{-\mathbf{A}_{Y}^{*}\right\}-\mathbf{N}_{Y}\right)$ is lc and that the set of lc centers of $\left(Y,\left\{-\mathbf{A}_{Y}^{*}\right\}-\mathbf{N}_{Y}\right)$ coincides with that of $\left(Y,-\mathbf{A}_{Y}^{*}-\mathbf{N}_{Y}\right)=\left(Y,-\mathbf{A}_{Y}\right)$. Therefore, the round-up of $\mathbf{A}_{Y^{\prime}}-g^{*}\left\lceil\mathbf{A}_{Y}^{*}\right\rceil-\mathbf{N}_{Y^{\prime}}$ is effective and $g$-exceptional. Thus, we can write $\left\lceil\mathbf{A}_{Y^{\prime}}^{*}\right\rceil=$ $g^{*}\left\lceil\mathbf{A}_{Y}^{*}\right\rceil+F$, where $F$ is a $g$-exceptional effective divisor.

The next lemma is obvious by Lemma 3.19.

Lemma 3.21. Let $(X, B)$ be a sub-lc pair and let $f: Y \rightarrow X$ be a proper birational morphism from a normal variety $Y$. We put $K_{Y}+B_{Y}=f^{*}\left(K_{X}+B\right)$. Then $f_{*} \mathcal{O}_{Y}\left(\left\lceil\mathbf{A}^{*}\left(Y, B_{Y}\right)\right\rceil\right)=\mathscr{O}_{X}\left(\left\lceil\mathbf{A}^{*}(X, B)\right\rceil\right)$.

\section{Basepoint-free theorem; nef and abundant case}

We recall the definition of abundant divisors, which are called good divisors in [Kawamata 1985]. See [Kawamata et al. 1987, Section 6-1].

Definition 4.1 (Abundant divisor). Let $X$ be a complete normal variety and let $D$ be a $\mathbb{Q}$-Cartier nef $\mathbb{Q}$-divisor on $X$. We define the numerical Iitaka dimension to be

$$
v(X, D)=\max \left\{e ; D^{e} \not \equiv 0\right\} .
$$

This means that $D^{e^{\prime}} \cdot S=0$ for any $e^{\prime}$-dimensional subvarieties $S$ of $X$ with $e^{\prime}>e$ and there exists an $e$-dimensional subvariety $T$ of $X$ such that $D^{e} \cdot T>0$. Then it is easy to see that $\kappa(X, D) \leq v(X, D)$, where $\kappa(X, D)$ denotes Iitaka's $D$-dimension. A nef $\mathbb{Q}$-divisor $D$ is said to be abundant if the equality $\kappa(X, D)=v(X, D)$ holds. Let $\pi: X \rightarrow S$ be a proper surjective morphism of normal varieties and let $D$ be a $\mathbb{Q}$-Cartier $\mathbb{Q}$-divisor on $X$. Then $D$ is said to be $\pi$-abundant if $\left.D\right|_{X_{\eta}}$ is abundant, where $X_{\eta}$ is the generic fiber of $\pi$.

The next theorem is the main theorem of [Kawamata 1985]. For the relative statement, see [Nakayama 1986, Theorem 5]. We reduced Theorem 4.2 to Theorem 2.1 by using Ambro's results in [Ambro 2004] and [Ambro 2007], which is the main theme of [Fujino 2011d]. For the details, see [Fujino 2011d, Section 2].

Theorem 4.2 cf. [Kawamata et al. 1987, Theorem 6-1-11]. Let $(X, B)$ be a klt pair and let $\pi: X \rightarrow S$ be a proper morphism onto a variety $S$. Assume the following conditions:

(a) $H$ is a $\pi$-nef $\mathbb{Q}$-Cartier $\mathbb{Q}$-divisor on $X$.

(b) $H-\left(K_{X}+B\right)$ is $\pi$-nef and $\pi$-abundant.

(c) $\kappa\left(X_{\eta},\left(a H-\left(K_{X}+B\right)\right)_{\eta}\right) \geq 0$ and

$$
v\left(X_{\eta},\left(a H-\left(K_{X}+B\right)\right)_{\eta}\right)=v\left(X_{\eta},\left(H-\left(K_{X}+B\right)\right)_{\eta}\right)
$$

for some $a \in \mathbb{Q}$ with $a>1$, where $\eta$ is the generic point of $S$.

Then $H$ is $\pi$-semiample. 
Definition 4.3 (Iitaka fibration). Let $\pi: X \rightarrow S$ be a proper surjective morphism of normal varieties. Let $D$ be a $\mathbb{Q}$-Cartier $\mathbb{Q}$-Weil divisor on $X$ such that $\kappa\left(X_{\eta}, D_{\eta}\right) \geq 0$, where $\eta$ is the generic point of $S$. Let $X \rightarrow W$ be the rational map over $S$ induced by $\pi^{*} \pi_{*} O_{X}(m D) \rightarrow \mathrm{O}_{X}(m D)$ for a sufficiently large and divisible integer $m$. We consider a projective surjective morphism $f: Y \rightarrow Z$ of nonsingular varieties that is birational to $X \rightarrow W$. We call $f: Y \rightarrow Z$ the Iitaka fibration with respect to $D$ over $S$.

We now state the main result of this section, which will be used in the proof of Theorem 7.11. It is a slight generalization of Theorem 4.2.

Theorem 4.4. Let $(X, B)$ be a sub-klt pair and let $\pi: X \rightarrow S$ be a proper morphism onto a variety $S$. Assume the following conditions:

(a) $H$ is a $\pi$-nef $\mathbb{Q}$-Cartier $\mathbb{Q}$-divisor on $X$.

(b) $H-\left(K_{X}+B\right)$ is $\pi$-nef and $\pi$-abundant.

(c) $\kappa\left(X_{\eta},\left(a H-\left(K_{X}+B\right)\right)_{\eta}\right) \geq 0$ and

$$
v\left(X_{\eta},\left(a H-\left(K_{X}+B\right)\right)_{\eta}\right)=v\left(X_{\eta},\left(H-\left(K_{X}+B\right)\right)_{\eta}\right)
$$

for some $a \in \mathbb{Q}$ with $a>1$, where $\eta$ is the generic point of $S$.

(d) Let $f: Y \rightarrow Z$ be the Iitaka fibration with respect to $H-\left(K_{X}+B\right)$ over $S$. We assume that there exists a proper birational morphism $\mu: Y \rightarrow X$ and put $K_{Y}+B_{Y}=\mu^{*}\left(K_{X}+B\right)$. In this setting, we assume rank $f_{*} \mathrm{O}_{Y}\left(\left\lceil\mathbf{A}\left(Y, B_{Y}\right)\right\rceil\right)=1$.

(e) (Saturation condition.) There exist positive integers $b$ and $j_{0}$ such that $b H$ is Cartier and $\pi_{*} \hat{O}_{X}(\lceil\mathbf{A}(X, B)\rceil+j b \bar{H}) \subseteq \pi_{*} \mathrm{O}_{X}(j b H)$ for every positive integer $j \geq j_{0}$.

Then $H$ is $\pi$-semiample.

Proof. The proof of Theorem 4.2 given in [Fujino 2011d, Section 2] works without any changes. We note that condition (d) implies [ibid., Lemma 2.3] and that we can use condition (e) in the proof of [ibid., Lemma 2.4].

Remark 4.5. The rank of $f_{*} \mathrm{O}_{Y}\left(\left\lceil\mathbf{A}\left(Y, B_{Y}\right)\right\rceil\right)$ is a birational invariant for $f: Y \rightarrow Z$ by Lemma 3.11.

Remark 4.6. If $(X, B)$ is klt and $b H$ is Cartier, it is obvious that

$$
\pi_{*} \mathrm{O}_{X}(\lceil\mathbf{A}(X, B)\rceil+j b \bar{H}) \simeq \pi_{*} \mathrm{O}_{X}(j b H)
$$

for every positive integer $j$ (see Remark 3.16).

Remark 4.7. We can easily generalize Theorem 4.4 to varieties in class $\mathscr{C}$ by suitable modifications. For details, see [Fujino 2011d, Section 4].

The following examples help us understand condition (d). 
Example 4.8. Let $X=E$ be an elliptic curve and let $P \in X$ be a closed point. Take a general member $P_{1}+P_{2}+P_{3} \in|3 P|$. We put $B=\frac{1}{3}\left(P_{1}+P_{2}+P_{3}\right)-P$. Then $(X, B)$ is sub-klt and $K_{X}+B \sim_{\mathbb{Q}} 0$. In this case, $\mathcal{O}_{X}(\lceil\mathbf{A}(X, B)\rceil) \simeq \mathscr{O}_{X}(P)$ and $H^{0}\left(X, \mathcal{O}_{X}(\lceil\mathbf{A}(X, B)\rceil)\right) \simeq k$.

Example 4.9. Let $f: X=\mathbb{P}_{\mathbb{P}^{1}}\left(\mathbb{O}_{\mathbb{p}^{1}} \oplus \mathcal{O}_{\mathbb{P}^{1}}(1)\right) \rightarrow Z=\mathbb{P}^{1}$ be the Hirzebruch surface and let $C$ (resp. $E$ ) be the positive (resp. negative) section of $f$. We take a general member $B_{0} \in|5 C|$. Note that $|5 C|$ is a free linear system on $X$. We put $B=-\frac{1}{2} E+\frac{1}{2} B_{0}$ and consider the pair $(X, B)$. Then $(X, B)$ is sub-klt. We put $H=0$. Then $H$ is a nef Cartier divisor on $X$ and $a H-\left(K_{X}+B\right) \sim_{\mathbb{Q}} \frac{1}{2} F$ for every rational number $a$, where $F$ is a fiber of $f$. Therefore, $a H-\left(K_{X}+B\right)$ is nef and abundant for every rational number $a$. In this case, $\mathscr{O}_{X}(\lceil\mathbf{A}(X, B)\rceil) \simeq \mathcal{O}_{X}(E)$. Thus

$$
\begin{aligned}
H^{0}\left(X, \mathcal{O}_{X}(\lceil\mathbf{A}(X, B)\rceil+j \bar{H})\right) & \simeq H^{0}\left(X, \mathrm{O}_{X}(E)\right) \simeq k \\
& \simeq H^{0}\left(X, \mathrm{O}_{X}\right) \simeq H^{0}\left(X, \mathrm{O}_{X}(j H)\right)
\end{aligned}
$$

for every integer $j$. Therefore, $\pi: X \rightarrow$ Spec $k, H$, and $(X, B)$ satisfy conditions (a), (b), (c), and (e) in Theorem 4.4. However, (d) is not satisfied. In our case, it is easy to see that $f: X \rightarrow Z$ is the Iitaka fibration with respect to $H-\left(K_{X}+B\right)$. Since $f_{*} \mathrm{O}_{X}(\lceil\mathbf{A}(X, B)\rceil) \simeq f_{*} \widehat{O}_{X}(E)$, we have rank $f_{*} \widehat{O}_{X}(\lceil\mathbf{A}(X, B)\rceil)=2$.

Remark 4.10. In Theorem 4.4, assumptions (a)-(c) are the same as in Theorem 4.2. Condition (e) is indispensable by Example 2.3 for sub-klt pairs. By using the nonvanishing theorem for generalized normal crossing varieties in [Kawamata 1985, Theorem 5.1], which is the hardest part to prove in [Kawamata 1985], the semiampleness of $H$ seems to follow from conditions (a), (b), (c), and (e). However, we need (d) to apply Ambro's canonical bundle formula to the Iitaka fibration $f: Y \rightarrow Z$. See, for example, [Fujino 2011d, Section 3]. Unfortunately, as we saw in Example 4.9, condition (d) does not follow from the other assumptions. Anyway, condition (d) is automatically satisfied if $(X, B)$ is klt; see [Fujino 2011d, Lemma 2.3].

The following two examples show that the effective version of Theorem 4.2 does not necessarily hold. The first one is an obvious example.

Example 4.11. Let $X=E$ be an elliptic curve and let $m$ be an arbitrary positive integer. Then there is a Cartier divisor $H$ on $X$ such that $m H \sim 0$ and $l H \nsim 0$ for $0<l<m$. Therefore, the effective version of Theorem 4.2 does not necessarily hold.

The next one shows the reason why Theorem 2.4 does not imply the effective version of Theorem 4.2.

Example 4.12. Let $E$ be an elliptic curve and $G=\mathbb{Z} / m \mathbb{Z}=\langle\zeta\rangle$, where $\zeta$ is a primitive $m$-th root of unity. We take an $m$-torsion point $a \in E$. The cyclic group 
$G$ acts on $E \times \mathbb{P}^{1}$ as follows:

$$
E \times \mathbb{P}^{1} \ni\left(x,\left[X_{0}: X_{1}\right]\right) \mapsto\left(x+a,\left[\zeta X_{0}: X_{1}\right]\right) \in E \times \mathbb{P}^{1} .
$$

We put $X=\left(E \times \mathbb{P}^{1}\right) / G$. Then $X$ has a structure of elliptic surface $p: X \rightarrow \mathbb{P}^{1}$. In this setting,

$$
K_{X}=p^{*}\left(K_{\mathbb{P}^{1}}+\frac{m-1}{m}[0]+\frac{m-1}{m}[\infty]\right) .
$$

We put $H=p^{-1}(0)_{\text {red }}$. Then $H$ is a Cartier divisor on $X$. It is easy to see that $H$ is nef and $H-K_{X}$ is nef and abundant. Moreover, $\kappa\left(X, a H-K_{X}\right)=$ $v\left(X, a H-K_{X}\right)=1$ for every rational number $a>0$. It is obvious that $|m H|$ is free. However, $|l H|$ is not free for $0<l<m$. Thus, the effective version of Theorem 4.2 does not hold.

\section{Basepoint-free theorem of Reid-Fukuda type}

The following result is a reformulation of the main theorem of [Fujino 2000].

Theorem 5.1 (Basepoint-free theorem of Reid-Fukuda type). Let $X$ be a nonsingular variety and let $B$ be a $\mathbb{Q}$-divisor on $X$ such that $\operatorname{Supp} B$ is a simple normal crossing divisor and $(X, B)$ is sub-lc. Let $\pi: X \rightarrow S$ be a proper morphism onto $a$ variety $S$ and let $D$ be a $\pi$-nef Cartier divisor on $X$. Assume the following conditions:

(1) $r D-\left(K_{X}+B\right)$ is nef and log big over $S$ for some positive integer $r$.

(2) (Saturation condition.) There exists a positive integer $j_{0}$ such that

$$
\pi_{*} O_{X}\left(\left\lceil\mathbf{A}^{*}(X, B)\right\rceil+j \bar{D}\right) \subseteq \pi_{*} O_{X}(j D)
$$

for every integer $j \geq j_{0}$.

Then $m D$ is $\pi$-generated for every $m \gg 0$, that is, there exists a positive integer $m_{0}$ such that for every $m \geq m_{0}$ the natural homomorphism $\pi^{*} \pi_{*} \mathrm{O}_{X}(m D) \rightarrow \mathrm{O}_{X}(m D)$ is surjective.

Definition 5.2. Let $(X, B)$ be a sub-lc pair and let $\pi: X \rightarrow S$ be a proper morphism onto a variety $S$. Let $\mathscr{L}$ be a line bundle on $X$. We say that $\mathscr{L}$ is nef and $\log$ big over $S$ if and only if $\mathscr{L}$ is $\pi$-nef and $\pi$-big and the restriction $\left.\mathscr{L}\right|_{W}$ is big over $\pi(W)$ for every lc center $W$ of the pair $(X, B)$. A $\mathbb{Q}$-Cartier $\mathbb{Q}$-divisor $H$ on $X$ is said to be nef and $\log$ big over $S$ if and only if so is $\mathbb{O}_{X}(c H)$, where $c$ is a positive integer such that $c H$ is Cartier.

Proof of Theorem 5.1. We write $B=T+B_{+}-B_{-}$such that $T, B_{+}$, and $B_{-}$are effective divisors, they have no common irreducible components, $\left\lfloor B_{+}\right\rfloor=0$, and 
$\lfloor T\rfloor=T$. If $T=0$, then $(X, B)$ is sub-klt. So, theorem follows from Theorem 2.1. Thus, we assume $T \neq 0$. Let $T_{0}$ be an irreducible component of $T$. If $m \geq r$, then

$$
m D+\left\lceil B_{-}\right\rceil-T_{0}-\left(K_{X}+B+\left\lceil B_{-}\right\rceil-T_{0}\right)=m D-\left(K_{X}+B\right)
$$

is nef and $\log$ big over $S$ for the pair $\left(X, B+\left\lceil B_{-}\right\rceil-T_{0}\right)$. We note that $B+\left\lceil B_{-}\right\rceil-T_{0}$ is effective. Therefore, $R^{1} \pi_{*} O_{X}\left(\left\lceil B_{-}\right\rceil-T_{0}+m D\right)=0$ for $m \geq r$ by the vanishing theorem: Lemma 5.3. Thus, we obtain the following commutative diagram for $m \geq \max \left\{r, j_{0}\right\}$ :

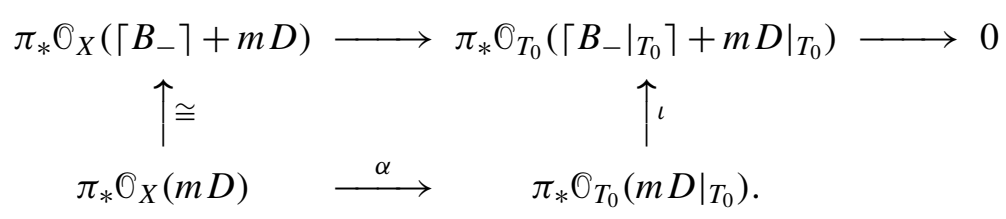

Here, we used

$$
\begin{aligned}
\pi_{*} O_{X}(m D) & \subseteq \pi_{*} O_{X}\left(\left\lceil B_{-}\right\rceil+m D\right) \\
& \simeq \pi_{*} O_{X}\left(\left\lceil\mathbf{A}^{*}(X, B)\right\rceil+m \bar{D}\right) \\
& \subseteq \pi_{*} O_{X}(m D)
\end{aligned}
$$

for $m \geq j_{0}$ (see Lemma 3.19). We put $K_{T_{0}}+B_{T_{0}}=\left.\left(K_{X}+B\right)\right|_{T_{0}}$ and $D_{T_{0}}=\left.D\right|_{T_{0}}$. Then $\left(T_{0}, B_{T_{0}}\right)$ is sub-lc and it is easy to see that $r D_{T_{0}}-\left(K_{T_{0}}+B_{T_{0}}\right)$ is nef and log big over $\pi\left(T_{0}\right)$. It is obvious that $T_{0}$ is nonsingular and Supp $B_{T_{0}}$ is a simple normal crossing divisor. We note that $\pi_{*} \mathcal{O}_{T_{0}}\left(\left\lceil\mathbf{A}^{*}\left(T_{0}, B_{T_{0}}\right)\right\rceil+j \overline{D_{T_{0}}}\right) \simeq \pi_{*} \mathrm{O}_{T_{0}}\left(j D_{T_{0}}\right)$ for every $j \geq \max \left\{r, j_{0}\right\}$ follows from the above diagram, that is, the natural inclusion $\iota$ is isomorphism for $m \geq \max \left\{r, j_{0}\right\}$. Thus, $\alpha$ is surjective for $m \geq \max \left\{r, j_{0}\right\}$. By induction, $m D_{T_{0}}$ is $\pi$-generated for every $m \gg 0$. We can apply the same argument to every irreducible component of $T$. Therefore, the relative base locus of $m D$ is disjoint from $T$ for every $m \gg 0$ since the restriction map $\alpha: \pi_{*} \mathscr{O}_{X}(m D) \rightarrow$ $\pi_{*} \mathrm{O}_{T_{0}}\left(m D_{T_{0}}\right)$ is surjective for every irreducible component $T_{0}$ of $T$. The arguments in [Fukuda 1996, Proof of Theorem 3], which is a variant of the X-method, work without any changes (cf. Theorem 6.1). So, we obtain that $m D$ is $\pi$-generated for every $m \gg 0$.

The following vanishing theorem was already used in the proof of Theorem 5.1. The proof is an easy exercise by induction on $\operatorname{dim} X$ and on the number of the irreducible components of $\lfloor\Delta\rfloor$.

Lemma 5.3. Let $\pi: X \rightarrow S$ be a proper morphism from a nonsingular variety $X$. Let $\Delta=\sum d_{i} \Delta_{i}$ be a sum of distinct prime divisors such that $\operatorname{Supp} \Delta$ is a simple normal crossing divisor and $d_{i}$ is a rational number with $0 \leq d_{i} \leq 1$ for every $i$. Let $D$ be a Cartier divisor on $X$. Assume that $D-\left(K_{X}+\Delta\right)$ is nef and log big over $S$ for the pair $(X, \Delta)$. Then $R^{i} \pi_{*} O_{X}(D)=0$ for every $i>0$. 
As in Theorem 2.4, effective freeness holds under the same assumption as in Theorem 5.1.

Theorem 5.4 (Effective freeness). We use the same notation and assumption as in Theorem 5.1. Then there exists a positive integer $l$, which depends only on $\operatorname{dim} X$ and $\max \left\{r, j_{0}\right\}$, such that $l D$ is $\pi$-generated, that is, $\pi^{*} \pi_{*} \mathscr{O}_{X}(l D) \rightarrow \mathrm{O}_{X}(l D)$ is surjective.

Sketch of the proof. If $(X, B)$ is sub-klt, then this theorem is nothing but Theorem 2.4. So, we can assume that $(X, B)$ is not sub-klt. In this case, the arguments in [Fukuda 1996, Section 4] work with only minor modifications. From now on, we use the notation in [Fukuda 1996, Section 4]. By minor modifications, the proof in [Fukuda 1996, Section 4] works under the following weaker assumptions: $X$ is nonsingular and $\Delta$ is a $\mathbb{Q}$-divisor on $X$ such that Supp $\Delta$ is a simple normal crossing divisor and $(X, \Delta)$ is sub-lc. In [Fukuda 1996, Claim 5], $E_{i}$ is $f$-exceptional. In our setting, this is not true. However, the bound

$$
0 \leq \sum_{c b_{i}-e_{i}+p_{i}<0}\left\lceil-\left(c b_{i}-e_{i}+p_{i}\right)\right\rceil E_{i} \leq\left\lceil\mathbf{A}^{*}(X, \Delta)_{Y}\right\rceil,
$$

which always holds even when $\Delta$ is not effective, is sufficient for us. It is because we can use the saturation condition (2) in Theorem 5.1. We leave the details as an exercise for the reader since all we have to do is to repeat the arguments in [Kollár 1993, Section 2] and [Fukuda 1996, Section 4].

The final statement in this section is the (effective) basepoint-free theorem of Reid-Fukuda type for dlt pairs.

Corollary 5.5. Let $(X, B)$ be a dlt pair and let $\pi: X \rightarrow S$ be a proper morphism onto a variety $S$. Let $D$ be a $\pi$-nef Cartier divisor on $X$. Assume that $r D-\left(K_{X}+B\right)$ is nef and log big over $S$ for some positive integer $r$. Then there exists a positive integer $m_{0}$ such that $m D$ is $\pi$-generated for every $m \geq m_{0}$ and we can find a positive integer $l$, which depends only on $\operatorname{dim} X$ and $r$, such that $l D$ is $\pi$-generated.

Proof. Let $f: Y \rightarrow X$ be a resolution such that $\operatorname{Exc}(f)$ and $\operatorname{Exc}(f) \cup \operatorname{Supp} f_{*}^{-1} B$ are simple normal crossing divisors, $K_{Y}+B_{Y}=f^{*}\left(K_{X}+B\right)$, and $f$ is an isomorphism over all the generic points of lc centers of the pair $(X, B)$. Then $\left(Y, B_{Y}\right)$ is sub-lc, and $r D_{Y}-\left(K_{Y}+B_{Y}\right)$ is nef and $\log$ big over $S$, where $D_{Y}=f^{*} D$. Since $\left\lceil\mathbf{A}^{*}(X, B)\right\rceil$ is effective and exceptional over $X, p_{*} \mathrm{O}_{Y}\left(\left\lceil\mathbf{A}^{*}\left(Y, B_{Y}\right)\right\rceil+j \overline{D_{Y}}\right) \simeq p_{*} \widehat{O}_{Y}\left(j D_{Y}\right)$ for every $j$, where $p=\pi \circ f$. So, we can apply Theorems 5.1 and 5.4 to $D_{Y}$ and $\left(Y, B_{Y}\right)$. This concludes the proof.

For the (effective) basepoint-freeness for lc pairs, see [Fujino 2009a; Fujino 2009b, 3.3.1 Base Point Free Theorem; Fujino 2011a, Theorem 13.1; Fujino 2011c, Theorem 1.2]. 


\section{Variants of basepoint-free theorems due to Fukuda}

The starting point of this section is a slight generalization of Theorem 2.1. It is essentially the same as [Fukuda 1996, Theorem 3].

Theorem 6.1. Let $X$ be a nonsingular variety and let $B$ be $a \mathbb{Q}$-divisor on $X$ such that $(X, B)$ is sub-lc and Supp $B$ is a simple normal crossing divisor. Let $\pi: X \rightarrow S$ be a proper morphism onto a variety $S$ and let $H$ be a $\pi$-nef $\mathbb{Q}$-Cartier $\mathbb{Q}$-divisor on $X$. Assume the following conditions:

(1) $H-\left(K_{X}+B\right)$ is nef and big over $S$.

(2) (Saturation condition.) There exist positive integers $b$ and $j_{0}$ such that

$$
\pi_{*} \mathrm{O}_{X}\left(\left\lceil\mathbf{A}^{*}(X, B)\right\rceil+j b \bar{H}\right) \subseteq \pi_{*} \mathbb{O}_{X}(j b H)
$$

for every integer $j \geq j_{0}$.

(3) There is a positive integer c such that $\mathrm{cH}$ is Cartier and

$$
\mathrm{O}_{T}(c H):=\left.\mathrm{O}_{X}(c H)\right|_{T}
$$

is $\pi$-generated, where $T=-\mathbf{N}(X, B)_{X}$.

Then $H$ is $\pi$-semiample.

Proof. If $(X, B)$ is sub-klt, then this follows from Theorem 2.1. By replacing $H$ by a multiple, we can assume that $b=1, j_{0}=1$, and $c=1$. Since

$$
l H+\left\lceil\mathbf{A}_{X}^{*}\right\rceil-T-\left(K_{X}+\{B\}\right)=l H-\left(K_{X}+B\right)
$$

is nef and big over $S$ for every positive integer $l$, we have the following commutative diagram by the Kawamata-Viehweg vanishing theorem:

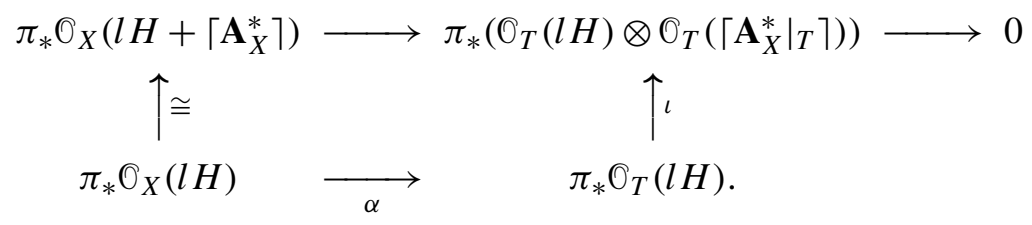

Thus, the natural inclusion $\iota$ is an isomorphism and $\alpha$ is surjective for every $l \geq 1$. In particular, $\pi_{*} \mathrm{O}_{X}(l H) \neq 0$ for every $l \geq 1$. The same arguments as in [Fukuda 1996, Proof of Theorem 3] show that $H$ is $\pi$-semiample.

The main purpose of this section is to prove Theorem 6.2 below, which is a generalization of Theorem 4.4 and Theorem 6.1. The basic strategy of the proof is the same as that of Theorem 4.4. That is, by using Ambro's canonical bundle formula, we reduce it to the case when $H-\left(K_{X}+B\right)$ is nef and big. This is nothing but Theorem 6.1. 
Theorem 6.2. Let $X$ be a nonsingular variety and let $B$ be $a \mathbb{Q}$-divisor on $X$ such that $(X, B)$ is sub-lc and Supp $B$ is a simple normal crossing divisor. Let $\pi: X \rightarrow S$ be a proper morphism onto a variety $S$. Assume the following conditions:

(a) $H$ is a $\pi$-nef $\mathbb{Q}$-Cartier $\mathbb{Q}$-divisor on $X$.

(b) $H-\left(K_{X}+B\right)$ is $\pi$-nef and $\pi$-abundant.

(c) $\kappa\left(X_{\eta},\left(a H-\left(K_{X}+B\right)\right)_{\eta}\right) \geq 0$ and $v\left(X_{\eta},\left(a H-\left(K_{X}+B\right)\right)_{\eta}\right)=v\left(X_{\eta},(H-\right.$ $\left.\left.\left(K_{X}+B\right)\right)_{\eta}\right)$ for some $a \in \mathbb{Q}$ with $a>1$, where $\eta$ is the generic point of $S$.

(d) Let $f: Y \rightarrow Z$ be the Iitaka fibration with respect to $H-\left(K_{X}+B\right)$ over $S$. We assume that there exists a proper birational morphism $\mu: Y \rightarrow X$ and put $K_{Y}+B_{Y}=\mu^{*}\left(K_{X}+B\right)$. We also assume rank $f_{*} \mathcal{O}_{Y}\left(\left\lceil\mathbf{A}^{*}\left(Y, B_{Y}\right)\right\rceil\right)=1$.

(e) (Saturation condition.) There exist positive integers $b$ and $j_{0}$ such that $b H$ is Cartier and $\pi_{*} \mathrm{O}_{X}\left(\left\lceil\mathbf{A}^{*}(X, B)\right\rceil+j b \bar{H}\right) \subseteq \pi_{*} \mathrm{O}_{X}(j b H)$ for every positive integer $j \geq j_{0}$.

(f) There is a positive integer $c$ such that $\mathrm{cH}$ is Cartier and

$$
\mathrm{O}_{T}(c H):=\left.\mathrm{O}_{X}(c H)\right|_{T}
$$

is $\pi$-generated, where $T=-\mathbf{N}(X, B)_{X}$.

Then $H$ is $\pi$-semiample.

Proof. If $H-\left(K_{X}+B\right)$ is big, this follows from Theorem 6.1. So, we can assume that $H-\left(K_{X}+B\right)$ is not big. Form now on, we use the notation from the proof of Theorem 4.2, which is given in [Fujino 2011d, Section 2]. We just explain how to modify that proof. Let us recall the commutative diagram

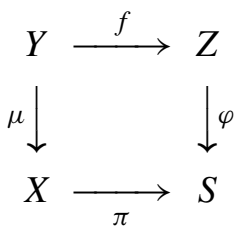

from the proof of [Fujino 2011d, Theorem 1.1], where $f: Y \rightarrow Z$ is the litaka fibration with respect to $H-\left(K_{X}+B\right)$ over $S$. For the details, see [Fujino 2011d, Section 2]. We note that $\mu^{*} H=H_{Y}$ and $H_{Y} \sim f^{*} D$. Here, we replaced $H$ with a multiple and assumed that $H$ and $D$ are Cartier (see [Fujino 2011d, page 307]). We can also assume that $b=j_{0}=1$ in (e) and $c=1$ in (f) by replacing $H$ with a multiple. We start with the following obvious lemma.

Lemma 6.3. We put $T^{\prime}=-\mathbf{N}(X, B)_{Y}$. Then $\mu\left(T^{\prime}\right) \subset T$. Therefore, $O_{T^{\prime}}\left(H_{Y}\right):=$ $\left.\mathrm{O}_{Y}\left(H_{Y}\right)\right|_{T^{\prime}}$ is p-generated, where $p=\pi \circ \mu$.

Lemma 6.4. If $f\left(T^{\prime}\right)=Z$, then $H_{Y}$ is p-semiample. In particular, $H$ is $\pi$ semiample. 
Proof. There exists an irreducible component $T_{0}^{\prime}$ of $T^{\prime}$ such that $f\left(T_{0}^{\prime}\right)=Z$. Since $\left.\left.\left(H_{Y}\right)\right|_{T_{0}^{\prime}} \sim\left(f^{*} D\right)\right|_{T_{0}^{\prime}}$ is $p$-semiample, $D$ is $\varphi$-semiample. This implies that $H_{Y}$ is $p$-semiample and $H$ is $\pi$-semiample.

Therefore, we can assume that $T^{\prime}$ is not dominant onto $Z$. Thus $\mathbf{A}\left(Y, B_{Y}\right)=$ $\mathbf{A}^{*}\left(Y, B_{Y}\right)$ over the generic point of $Z$. Equivalently, $\left(Y, B_{Y}\right)$ is sub-klt over the generic point of $Z$. As in [Fujino 2011d, Proof of Theorem 1.1], we have these properties:

(1) $K_{Y}+B_{Y} \sim_{\mathbb{Q}} f^{*}\left(K_{Z}+B_{Z}+M\right)$, where $B_{Z}$ is the discriminant $\mathbb{Q}$-divisor of $\left(Y, B_{Y}\right)$ on $Z$ and $M$ is the moduli $\mathbb{Q}$-divisor on $Z$.

( $\left.2^{\prime}\right)\left(Z, B_{Z}\right)$ is sub-lc.

(3) $M$ is a $\varphi$-nef $\mathbb{Q}$-divisor on $Z$.

(4') $\varphi_{*} \mathrm{O}_{Z}\left(\left\lceil\mathbf{A}^{*}\left(Z, B_{Z}\right)\right\rceil+j \bar{D}\right) \subseteq \varphi_{*} \mathcal{O}_{Z}(j D)$ for every positive integer $j$.

(5) $D-\left(K_{Z}+B_{Z}\right)$ is $\varphi$-nef and $\varphi$-big.

(6) $Y$ and $Z$ are nonsingular and Supp $B_{Y}$ and Supp $B_{Z}$ are simple normal crossing divisors.

(7) $\mathcal{O}_{T^{\prime \prime}}(D):=\left.\mathscr{O}_{Z}(D)\right|_{T^{\prime \prime}}$ is $\varphi$-generated where $T^{\prime \prime}=-\mathbf{N}\left(Z, B_{Z}\right)_{Z}$.

Once the conditions above were satisfied, $D$ is $\varphi$-semiample by Theorem 6.1 . Therefore, $H$ is $\pi$-semiample. So, all we have to do is check the conditions. Conditions (1), (2'), (3), (5), (6) are satisfied by a result of Ambro; see [Fujino 2011d, Proof of Theorem 1.1]. We note that $f_{*} \mathrm{O}_{Y}\left(\left\lceil\mathbf{A}\left(Y, B_{Y}\right)\right\rceil\right)$ and $f_{*} \mathcal{O}_{Y}\left(\left\lceil\mathbf{A}^{*}\left(Y, B_{Y}\right)\right\rceil\right)$ both have rank 1. By the same computation as in [Ambro 2007, Lemma 9.2.2 and Proposition 9.2.3], we have the following lemma.

Lemma 6.5. $\mathrm{O}_{Z}\left(\left\lceil\mathbf{A}^{*}\left(Z, B_{Z}\right)\right\rceil+j \bar{D}\right) \subseteq f_{*} \widehat{O}_{Y}\left(\left\lceil\mathbf{A}^{*}\left(Y, B_{Y}\right)\right\rceil+j \overline{H_{Y}}\right)$ for every integer $j$.

Thus, we have (4') by the saturation condition (e) (for details, see [Fujino 2011d, Proof of Theorem 1.1], and Lemma 3.21). By definition, we have

$$
l H_{Y}+\left\lceil\mathbf{A}_{Y}^{*}\right\rceil-T^{\prime}-\left(K_{Y}+\left\{B_{Y}\right\}\right) \sim_{\mathbb{Q}} f^{*}\left((l-1) D+M_{0}\right),
$$

where

$$
H_{Y}-\left(K_{Y}+B_{Y}\right)=\mu^{*}\left(H-\left(K_{X}+B\right)\right) \sim_{\mathbb{Q}} f^{*} M_{0} .
$$

Note that $(l-1) D+M_{0}$ is $\varphi$-nef and $\varphi$-big for $l \geq 1$. By the Kollár type injectivity theorem,

$$
R^{1} p_{*} \mathrm{O}_{Y}\left(l H_{Y}+\left\lceil\mathbf{A}_{Y}^{*}\right\rceil-T^{\prime}\right) \rightarrow R^{1} p_{*} \widehat{O}_{Y}\left(l H_{Y}+\left\lceil\mathbf{A}_{Y}^{*}\right\rceil\right)
$$

is injective for $l \geq 1$. Note that the above injectivity can be checked easily by [Fujino 2007a, Theorem 1.1]. Here, we used the fact that $f\left(T^{\prime}\right) \subsetneq Z$. So, we have 
the commutative diagram

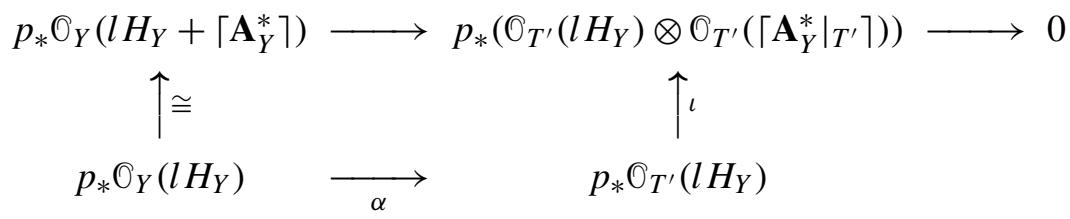

The isomorphism of the left vertical arrow follows from the saturation condition (e). Thus, the natural inclusion $\iota$ is an isomorphism and $\alpha$ is surjective for $l \geq 1$. In particular, the relative base locus of $l H_{Y}$ is disjoint from $T^{\prime}$ since $O_{T^{\prime}}\left(l H_{Y}\right)$ is $p$-generated (cf. Lemma 6.3). On the other hand, $H_{Y} \sim f^{*} D$. Therefore, $\mathrm{O}_{T^{\prime \prime}}(D)$ is $\varphi$-generated since $T^{\prime \prime} \subset f\left(T^{\prime}\right)$. So, we obtain condition (7). This completes the proof of Theorem 6.2.

As a corollary of Theorem 6.2, we obtain the generalization of [Fukuda 2002, Proposition 3.3] stated in the introduction (Theorem 1.1). Before we derive it, we recall the definition of non-klt loci.

Definition 6.6 (Non-klt locus). Let $(X, B)$ be an lc pair. We consider the closed subset

$$
\operatorname{Nklt}(X, B)=\{x \in X \mid(X, B) \text { is not klt at } x\}
$$

of $X$. We call $\operatorname{Nklt}(X, B)$ the non-klt locus of $(X, B)$.

Proof of Theorem 1.1. Let $h: X^{\prime} \rightarrow X$ be a resolution such that $\operatorname{Exc}(h) \cup \operatorname{Supp} h_{*}^{-1} B$ is a simple normal crossing divisor and $K_{X^{\prime}}+B_{X^{\prime}}=h^{*}\left(K_{X}+B\right)$. Then $H_{X^{\prime}}=h^{*} H$, $\left(X^{\prime}, B_{X^{\prime}}\right.$ ), and $\pi^{\prime}=\pi \circ h: X^{\prime} \rightarrow S$ satisfy assumptions (a), (b), and (c) in Theorem 6.2. By the same argument as in the proof of [Fujino 2011d, Lemma 2.3], we obtain rank $f_{*} \mathcal{O}_{Y}\left(\left\lceil\mathbf{A}^{*}\left(Y, B_{Y}\right)\right\rceil\right)=1$, where $f: Y \rightarrow Z$ is the Iitaka fibration as in (d) in Theorem 6.2. Note that $\left\lceil\mathbf{A}^{*}\left(Y, B_{Y}\right)\right\rceil$ is effective and exceptional over $X$. Since $B$ is effective, $\left\lceil\mathbf{A}^{*}(X, B)\right\rceil$ is effective and exceptional over $X$,

$$
\pi_{*}^{\prime} \mathrm{O}_{X^{\prime}}\left(\left\lceil\mathbf{A}^{*}\left(X^{\prime}, B_{X^{\prime}}\right)\right\rceil+j b \overline{H_{X^{\prime}}}\right) \subseteq \pi_{*}^{\prime} \widehat{O}_{X^{\prime}}\left(j b H_{X^{\prime}}\right)
$$

for every integer $j$, where $b$ is a positive integer such that $b H$ is Cartier. So, the saturation condition (e) in Theorem 6.2 is satisfied. Finally, $O_{T^{\prime}}\left(c H_{X^{\prime}}\right):=$ $\left.\mathrm{O}_{X^{\prime}}\left(c H_{X^{\prime}}\right)\right|_{T^{\prime}}$ is $\pi^{\prime}$-generated, where $T^{\prime}=-\mathbf{N}(X, B)_{X^{\prime}}$, by assumption (D) and the fact that $h\left(T^{\prime}\right) \subset T$. So, condition (f) in Theorem 6.2 for $H_{X^{\prime}}$ and $\left(X^{\prime}, B_{X^{\prime}}\right)$ is satisfied. Therefore, $H_{X^{\prime}}$ is $\pi^{\prime}$-semiample by Theorem 6.2. Thus, $H$ is $\pi$ semiample.

Remark 6.7. (i) It is obvious that $\operatorname{Supp}\left(-\mathbf{N}(X, B)_{X}\right) \subseteq \operatorname{Nklt}(X, B)$. In general, $\operatorname{Supp}\left(-\mathbf{N}(X, B)_{X}\right) \subsetneq \operatorname{Nklt}(X, B)$. In particular, $\operatorname{Nklt}(X, B)$ is not necessarily of pure codimension one in $X$. 
(ii) If $(X, B)$ is dlt, then $\operatorname{Nklt}(X, B)=\operatorname{Supp}\left(-\mathbf{N}(X, B)_{X}\right)=\lfloor B\rfloor$. Therefore, if $(X, B)$ is dlt and $S$ is a point, then Theorem 1.1 is nothing but Fukuda's result [Fukuda 2002, Proposition 3.3].

(iii) The reader can find applications of this corollary in [Fukuda 2002; Fujino 2010; Fujino and Gongyo 2011].

By combining Theorem 1.1 with [Gongyo 2010, Theorem 1.5], we obtain the following result.

Corollary 6.8. Let $(X, B)$ be a projective dlt pair such that $v\left(K_{X}+B\right)=\kappa\left(K_{X}+B\right)$ and that $\left.\left(K_{X}+B\right)\right|_{\lfloor B\rfloor}$ is numerically trivial. Then $K_{X}+B$ is semiample.

Remark 6.9. We can easily generalize Theorem 6.2 and Theorem 1.1 to varieties in class $\mathscr{C}$ by suitable modifications. For details, see [Fujino 2011d, Section 4].

\section{Basepoint-free theorems for pseudo-klt pairs}

In this section, we generalize the Kawamata-Shokurov base point free theorem and Kawamata's theorem: Theorem 4.2 for klt pairs to pseudo-klt pairs. We think that our formulation is useful when we study lc centers (see Proposition 7.8). First, we introduce the notion of pseudo-klt pairs.

Definition 7.1 (Pseudo-klt pair). Let $W$ be a normal variety. Assume the following conditions:

(1) there exist a sub-klt pair $(V, B)$ and a projective surjective morphism $f: V \rightarrow W$ with connected fibers.

(2) $f_{*} \mathcal{O}_{V}(\lceil\mathbf{A}(V, B)\rceil) \simeq 0_{W}$.

(3) There exists a $\mathbb{Q}$-Cartier $\mathbb{Q}$-divisor $\mathscr{K}$ on $W$ such that $K_{V}+B \sim_{\mathbb{Q}} f^{*} \mathscr{K}$.

Then the pair $[W, \mathscr{K}]$ is called a pseudo-klt pair.

Although it is the first time that we use the name of pseudo-klt pair, the notion of pseudo-klt pair appeared in [Fujino 1999], where we proved the cone and contraction theorem for pseudo-klt pairs (cf. [Fujino 1999, Section 4]). We note that all the fundamental theorems for the log minimal model program for pseudo-klt pairs can be proved by the theory of quasilog varieties (cf. [Ambro 2003; Fujino 2009b; 2011b]).

Remark 7.2. In Definition 7.1, we assume that $W$ is normal. However, the normality of $W$ follows from condition (2) and the normality of $V$. Note that $\lceil\mathbf{A}(V, B)\rceil$ is effective.

Remark 7.3. In the definition of pseudo-klt pairs, if $(V, B)$ is klt, the condition $f_{*} \mathcal{O}_{V}(\lceil\mathbf{A}(V, B)\rceil) \simeq \mathfrak{O}_{W}$ is automatically satisfied. This is because $\lceil\mathbf{A}(V, B)\rceil$ is effective and exceptional over $V$. 
We note that a pseudo-klt pair is a very special example of Ambro's quasilog varieties (see [Ambro 2003, Definition 4.1]). More precisely, if $[V, \mathscr{K}]$ is a pseudoklt pair, then we can easily check that $[V, \mathscr{K}]$ is a qlc pair. See, for example, [Fujino 2011b, Definition 3.1]. For the details of the theory of quasilog varieties, see [Fujino 2009b].

Theorem 7.4. Let $[W, \mathscr{K}]$ be a pseudo-klt pair. Assume that $(V, B)$ is klt and $W$ is projective or that $W$ is affine. Then we can find an effective $\mathbb{Q}$-divisor $B_{W}$ on $W$ such that $\left(W, B_{W}\right)$ is klt and that $\mathscr{N} \sim_{\mathbb{Q}} K_{W}+B_{W}$.

Proof. When $(X, B)$ is klt and $W$ is projective, we can find $B_{W}$ by [Ambro 2005a, Theorem 4.1]. When $W$ is affine, this theorem follows from [Fujino 1999, Theorem 1.2].

Remark 7.5. It is conjectured that one can always find an effective $\mathbb{Q}$-divisor $B_{W}$ on $W$ such that $\left(W, B_{W}\right)$ is klt and $\mathscr{K} \sim_{\mathbb{Q}} K_{W}+B_{W}$.

We now collect basic examples of pseudo-klt pairs.

Example 7.6. A klt pair is a pseudo-klt pair.

Example 7.7. Let $f: X \rightarrow W$ be a Mori fiber space. Then we can find a $\mathbb{Q}$-Cartier $\mathbb{Q}$-divisor $\mathscr{K}$ on $W$ such that $[W, \mathscr{K}]$ is a pseudo-klt pair. It is because we can find an effective $\mathbb{Q}$-divisor $B$ on $X$ such that $K_{X}+B \sim_{\mathbb{Q}, f} 0$ and $(X, B)$ is klt.

Proposition 7.8. An exceptional lc center $W$ of an lc pair $(X, B)$ is a pseudo-klt pair for some $\mathbb{Q}$-Cartier $\mathbb{Q}$-divisor $\%$ on $W$.

Proof. We take a resolution $g: Y \rightarrow X$ such that $\operatorname{Exc}(g) \cup g_{*}^{-1} B$ has a simple normal crossing support. We put $K_{Y}+B_{Y}=g^{*}\left(K_{X}+B\right)$. Then $-B_{Y}=\mathbf{A}(X, B)_{Y}=\mathbf{A}_{Y}=$ $\mathbf{A}_{Y}^{*}+\mathbf{N}_{Y}$, where $\mathbf{N}_{Y}=-\sum_{i=0}^{k} E_{i}$. Without loss of generality, we can assume that $f(E)=W$ and $E=E_{0}$. By shrinking $X$ around $W$, we can assume that $\mathbf{N}_{Y}=-E$. Note that $R^{1} g_{*} \mathrm{O}_{Y}\left(\left\lceil\mathbf{A}_{Y}^{*}\right\rceil-E\right)=0$ by the Kawamata-Viehweg vanishing theorem since $\left\lceil\mathbf{A}_{Y}^{*}\right\rceil-E=K_{Y}+\left\{-\mathbf{A}_{Y}^{*}\right\}-g^{*}\left(K_{X}+B\right)$. Therefore, $g_{*} \mathcal{O}_{Y}\left(\left\lceil\mathbf{A}_{Y}^{*}\right\rceil\right) \simeq \mathfrak{O}_{X} \rightarrow$ $g_{*} \mathrm{O}_{E}\left(\left\lceil\left.\mathbf{A}_{Y}^{*}\right|_{E}\right\rceil\right)$ is surjective. This implies that $g_{*} \mathcal{O}_{E}\left(\left\lceil\left.\mathbf{A}_{Y}^{*}\right|_{E}\right\rceil\right) \simeq \mathcal{O}_{W}$. In particular, $W$ is normal. If we put $K_{E}+B_{E}=\left.\left(K_{Y}+B_{Y}\right)\right|_{E}$, then $\left(E, B_{E}\right)$ is sub-klt and $\left.\mathbf{A}_{Y}^{*}\right|_{E}=\mathbf{A}\left(E, B_{E}\right)_{E}=-B_{E}$. So, $g_{*} \mathscr{O}_{E}\left(\left\lceil\mathbf{A}\left(E, B_{E}\right)\right\rceil\right)=g_{*} \widehat{O}_{E}\left(\left\lceil-B_{E}\right\rceil\right) \simeq \mathfrak{O}_{W}$. Since $K_{E}+B_{E}=\left.\left(K_{Y}+B_{Y}\right)\right|_{E}$ and $K_{Y}+B_{Y}=g^{*}\left(K_{X}+B\right)$, we can find a $\mathbb{Q}$-Cartier $\mathbb{Q}$-divisor $\mathscr{K}$ on $W$ such that $K_{E}+B_{E} \sim_{\mathbb{Q}} g^{*} \mathscr{K}$. Therefore, $W$ is a pseudo-klt pair.

We make an important remark on minimal lc centers.

Remark 7.9 (Subadjunction for minimal lc center). Let $(X, B)$ be a projective or affine lc pair and let $W$ be a minimal lc center of the pair $(X, B)$. Then we can find an effective $\mathbb{Q}$-divisor $B_{W}$ on $W$ such that $\left(W, B_{W}\right)$ is klt and $K_{W}+\left.B_{W} \sim_{\mathbb{Q}}\left(K_{X}+B\right)\right|_{W}$. For the details, see [Fujino and Gongyo 2012, Theorems 4.1, 7.1]. 
The following theorem is the Kawamata-Shokurov basepoint-free theorem for pseudo-klt pairs. We give a simple proof depending on Kawamata's positivity theorem. Although Theorem 7.10 seems to be contained in [Ambro 2003, Theorem 7.2], no proof is given there.

Theorem 7.10. Let $[W, \mathscr{K}]$ be a pseudo-klt pair, let $\pi: W \rightarrow S$ be a proper morphism onto a variety $S$ and let $D$ be a $\pi$-nef Cartier divisor on $W$. Assume that $r D-Y$ is $\pi$-nef and $\pi$-big for some positive integer $r$. Then $m D$ is $\pi$-generated for every $m \gg 0$.

Proof. Without loss of generality, we can assume that $S$ is affine. By the usual technique (see [Kawamata 1998, Theorem 1] and [Fujino 1999, Theorem 1.2]), we have

$$
\mathscr{K}+\varepsilon(r D-\mathscr{K}) \sim_{\mathbb{Q}} K_{W}+\Delta_{W}
$$

such that $\left(W, \Delta_{W}\right)$ is klt for some sufficiently small rational number $0<\varepsilon \ll 1$ (see also [Kollár 2007, Theorem 8.6.1]). Then $r D-\left(K_{W}+\Delta_{W}\right) \sim_{\mathbb{Q}}(1-\varepsilon)(r D-\mathscr{K})$, which is $\pi$-nef and $\pi$-big. Therefore, $m D$ is $\pi$-generated for every $m \gg 0$ by the usual Kawamata-Shokurov basepoint-free theorem.

The next theorem is the main theorem of this section. It is a generalization of Kawamata's theorem in [Kawamata 1985] (cf. Theorem 4.2) for pseudo-klt pairs.

Theorem 7.11. Let $[W, \mathscr{K}]$ be a pseudo-klt pair and let $\pi: W \rightarrow S$ be a proper morphism onto a variety $S$. Assume the following conditions:

(i) $H$ is a $\pi$-nef $\mathbb{Q}$-Cartier $\mathbb{Q}$-divisor on $W$.

(ii) $H-\mathscr{K}$ is $\pi$-nef and $\pi$-abundant.

(iii) $\kappa\left(W_{\eta},(a H-\mathscr{K})_{\eta}\right) \geq 0$ and $v\left(W_{\eta},(a H-\mathscr{K})_{\eta}\right)=v\left(W_{\eta},(H-\mathscr{K})_{\eta}\right)$ for some $a \in \mathbb{Q}$ with $a>1$, where $\eta$ is the generic point of $S$.

Then $H$ is $\pi$-semiample.

Proof. By definition, there exists a proper surjective morphism $f: V \rightarrow W$ from a sub-klt pair $(V, B)$. Without loss of generality, we can assume that $V$ is nonsingular and Supp $B$ is a simple normal crossing divisor. By definition, $f_{*} \mathcal{O}_{V}(\lceil-B\rceil) \simeq \mathfrak{O}_{W}$. From now on, we assume that $H$ is Cartier by replacing it with a multiple. Then $f_{*} \mathcal{O}_{V}\left(\lceil-B\rceil+j H_{V}\right) \simeq \mathfrak{O}_{W}(j H)$ by the projection formula for every integer $j$, where $H_{V}=f^{*} H$. Pushing forward by $\pi$, we have

$$
\begin{aligned}
p_{*} \mathscr{O}_{V}\left(\lceil\mathbf{A}(V, B)\rceil+j \overline{H_{V}}\right) & =p_{*} \mathscr{O}_{V}\left(\lceil-B\rceil+j H_{V}\right) \\
& \simeq \pi_{*} \mathbb{O}_{W}(j H) \\
& \simeq p_{*} \mathscr{O}_{V}\left(j H_{V}\right)
\end{aligned}
$$


for every integer $j$, where $p=\pi \circ f$. This is nothing but the saturation condition Theorem 4.4(e). We put $L=H-\mathscr{K}$. We consider the Iitaka fibration with respect to $L$ over $S$ as in [Fujino 2011d, Proof of Theorem 1.1]. Then we obtain the following commutative diagram:

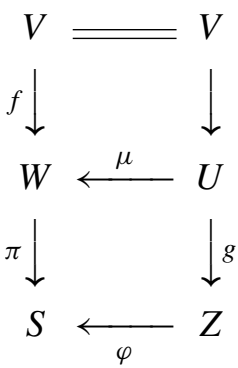

where $g: U \rightarrow Z$ is the Iitaka fibration over $S$ and $\mu: U \rightarrow W$ is a birational morphism. Note that we can assume that $f: V \rightarrow W$ factors through $U$ by blowing up $V$.

Lemma 7.12. $\operatorname{rank} h_{*} \mathcal{O}_{V}(\lceil\mathbf{A}(V, B)\rceil)=1$, where $h: V \rightarrow U \rightarrow Z$.

Proof. This proof is essentially the same as that of [Fujino 2011d, Lemma 2.3]. First, we can assume that $S$ is affine. Let $A$ be an ample divisor on $Z$ such that $h_{*} \mathcal{O}_{V}(\lceil\mathbf{A}(V, B)\rceil) \otimes \mathrm{O}_{Z}(A)$ is $\varphi$-generated. We note that we can assume that $\mu^{*} L \sim_{\mathbb{Q}} g^{*} M$ since $L$ is $\pi$-nef and $\pi$-abundant, where $M$ is a $\varphi$-nef and $\varphi$-big $\mathbb{Q}$ divisor on $Z$. If we choose a large and divisible integer $m$, then $\mathrm{O}_{Z}(A) \subset \mathrm{O}_{Z}(m M)$. Thus

$$
\begin{aligned}
& \varphi_{*}\left(h_{*} \mathrm{O}_{V}(\lceil\mathbf{A}(V, B)\rceil) \otimes \mathbb{O}_{Z}(A)\right) \\
\subseteq & \varphi_{*}\left(h_{*} \widehat{O}_{V}(\lceil\mathbf{A}(V, B)\rceil) \otimes \mathbb{O}_{Z}(m M)\right) \\
\simeq & p_{*} \mathscr{O}_{V}\left(\lceil\mathbf{A}(V, B)\rceil+m \overline{f^{*} L}\right) \\
\simeq & \pi_{*} \widehat{O}_{W}(m L) \\
\simeq & \varphi_{*} \mathbb{O}_{Z}(m M) .
\end{aligned}
$$

Therefore, $\operatorname{rank} h_{*} \mathcal{O}_{V}(\lceil\mathbf{A}(V, B)\rceil) \leq 1$. Since $\mathfrak{O}_{Z} \subset h_{*} \mathscr{O}_{V} \subset h_{*} \mathscr{O}_{V}(\lceil\mathbf{A}(V, B)\rceil)$, we obtain $\operatorname{rank} h_{*} \mathcal{O}_{V}(\lceil\mathbf{A}(V, B)\rceil)=1$

Note that $h: V \rightarrow Z$ is the Iitaka fibration with respect to $f^{*} L$ over $S$. Assumption (c) in Theorem 4.4 easily follows from (iii). Thus, by Theorem 4.4, we have that $H_{V}$ is $p$-semiample. Equivalently, $H$ is $\pi$-semiample.

The final theorem of this paper is a basepoint-free theorem for minimal lc centers. Theorem 7.13. Let $(X, B)$ be a quasi-projective lc pair and let $W$ be a minimal lc center of $(X, B)$. Let $\pi: W \rightarrow S$ be a proper morphism onto a variety $S$. Assume the following conditions: 
(i) $H$ is a $\pi$-nef $\mathbb{Q}$-Cartier $\mathbb{Q}$-divisor on $W$.

(ii) $H-\left.\left(K_{X}+B\right)\right|_{W}$ is $\pi$-nef and $\pi$-abundant.

(iii) $\kappa\left(W_{\eta},\left.\left(a H-\left(K_{X}+B\right)\right)\right|_{W_{\eta}}\right) \geq 0$ and

$$
v\left(W_{\eta},\left.\left(a H-\left(K_{X}+B\right)\right)\right|_{W_{\eta}}\right)=v\left(W_{\eta},\left.\left(H-\left(K_{X}+B\right)\right)\right|_{W_{\eta}}\right)
$$

for some $a \in \mathbb{Q}$ with $a>1$, where $\eta$ is the generic point of $S$.

Then $H$ is $\pi$-semiample.

Proof. Let $f: Y \rightarrow X$ be a dlt blow-up such that $K_{Y}+B_{Y}=f^{*}\left(K_{X}+B\right)$ (see, for example, [Fujino 2011a, Theorem 10.4] or [Fujino 2011e, Section 4]). Then we can take a minimal lc center $Z$ of $\left(Y, B_{Y}\right)$ such that $f(Z)=W$. Note that $K_{Z}+B_{Z}=\left.\left(K_{Y}+B_{Y}\right)\right|_{Z}$ is klt. We also note that $W$ is normal (see, for example, [Fujino 2011c, Theorem 2.4 (4)] or [Fujino 2011a, Theorem 9.1 (4)]). Let

$$
f: Z \stackrel{g}{\longrightarrow} V \stackrel{h}{\longrightarrow} W
$$

be the Stein factorization of $f: Z \rightarrow W$. Then $\left[V, h^{*}\left(\left.\left(K_{X}+B\right)\right|_{W}\right)\right]$ is a pseudo-klt pair by $g:\left(Z, B_{Z}\right) \rightarrow V$. We note that $H$ is $\pi$-semiample if and only if $h^{*} H$ is $\pi \circ h$-semiample. By Theorem $7.11, h^{*} H$ is semiample over $S$. This concludes the proof.

\section{Acknowledgement}

The first version of this paper was written in Nagoya in 2005 and posted on arXiv [Fujino 2005]. At the time, the author was partially supported by The Sumitomo Foundation and by the Grant-in-Aid for Young Scientists (A) 17684001 from JSPS. He revised this paper in Kyoto in 2011, where he was partially supported by the Inamori Foundation and by the Grant-in-Aid for Young Scientists (A) 20684001 from JSPS.

The author thanks the referee for comments.

\section{References}

[Ambro 2003] F. Ambro, “Quasi-log varieties”, Tr. Mat. Inst. Steklova 240 (2003), 220-239. Reprinted in Proc. Steklov Inst. Math. 240: (2003), 214-233. MR 2004f:14027 Zbl 1081.14021

[Ambro 2004] F. Ambro, "Shokurov's boundary property", J. Differential Geom. 67:2 (2004), 229255. In Russian; translated in Proc. Steklov Inst. Math. 240: (2003), 214-233. MR 2006d:14033 Zbl 1097.14029

[Ambro 2005a] F. Ambro, "The moduli b-divisor of an lc-trivial fibration", Compos. Math. 141:2 (2005), 385-403. MR 2006d:14015 Zbl 1094.14025

[Ambro 2005b] F. Ambro, “A semiampleness criterion”, J. Math. Sci. Univ. Tokyo 12:3 (2005), 445-466. MR 2007i:14018 Zbl 1121.14005 
[Ambro 2007] F. Ambro, "Non-klt techniques", pp. 163-170 in Flips for 3-folds and 4-folds, edited by A. Corti, Oxford Lecture Ser. Math. Appl. 35, Oxford Univ. Press, 2007. MR 2359347

[Cacciola 2011] S. Cacciola, "On the semiampleness of the positive part of CKM Zariski decompositions”, preprint, 2011. arXiv 1011.6564v2

[Corti 2007] A. Corti, "3-fold flips after Shokurov", pp. 18-48 in Flips for 3-folds and 4-folds, edited by A. Corti, Oxford Lecture Ser. Math. Appl. 35, Oxford Univ. Press, 2007. MR 2359339

[Corti et al. 2007] A. Corti (editor), Flips for 3-folds and 4-folds, Oxford Lecture Ser. Math. Appl. 35, Oxford Univ. Press, 2007. MR 2359340

[Fujino 1999] O. Fujino, "Applications of Kawamata's positivity theorem", Proc. Japan Acad. Ser. A Math. Sci. 75:6 (1999), 75-79. MR 2000f:14089 Zbl 0967.14012

[Fujino 2000] O. Fujino, "Base point free theorem of Reid-Fukuda type", J. Math. Sci. Univ. Tokyo 7:1 (2000), 1-5. MR 2001c:14013 Zbl 0971.14009

[Fujino 2003] O. Fujino, "A canonical bundle formula for certain algebraic fiber spaces and its applications”, Nagoya Math. J. 172 (2003), 129-171. MR 2005b:14024 Zbl 1072.14040

[Fujino 2005] O. Fujino, "A remark on the basepoint-free theorem”, preprint, 2005. arXiv 0508.554v1

[Fujino 2007a] O. Fujino, “A transcendental approach to Kollár's injectivity theorem II”, preprint, 2007. To appear in J. reine angew. math. arXiv 0705.1199v1

[Fujino 2007b] O. Fujino, "What is log terminal?", pp. 49-62 in Flips for 3-folds and 4-folds, edited by A. Corti, Oxford Lecture Ser. Math. Appl. 35, Oxford Univ. Press, 2007. MR 2359341

[Fujino 2009a] O. Fujino, "Effective base point free theorem for log canonical pairs-Kollár type theorem", Tohoku Math. J., (2) 61:4 (2009), 475-481. MR 2011c:14014 Zbl 1189.14025

[Fujino 2009b] O. Fujino, "Introduction to the log minimal model program for log canonical pairs", preprint, 2009. arXiv 0907.1506v1

[Fujino 2009c] O. Fujino, "On injectivity, vanishing and torsion-free theorems for algebraic varieties", Proc. Japan Acad. Ser. A Math. Sci. 85:8 (2009), 95-100. MR 2011c:14053 Zbl 1189.14024

[Fujino 2010] O. Fujino, "Finite generation of the log canonical ring in dimension four", Kyoto J. Math. 50:4 (2010), 671-684. MR 2012c:14032 Zbl 1210.14020

[Fujino 2011a] O. Fujino, "Fundamental theorems for the log minimal model program", Publ. Res. Inst. Math. Sci. 47:3 (2011), 727-789. MR 2832805 Zbl 1234.14013

[Fujino 2011b] O. Fujino, "Introduction to the theory of quasi-log varieties", pp. 289-303 in Classification of algebraic varieties, edited by C. Faber et al., Eur. Math. Soc., Zürich, 2011. MR 2012f:14025 Zbl 1213.14030

[Fujino 2011c] O. Fujino, "Non-vanishing theorem for log canonical pairs", J. Algebraic Geom. 20:4 (2011), 771-783. MR 2819675 Zbl 05955440

[Fujino 2011d] O. Fujino, “On Kawamata's theorem”, pp. 305-315 in Classification of algebraic varieties, edited by C. Faber et al., Eur. Math. Soc., Zürich, 2011. MR 2012c:14011 Zbl 1213.14015

[Fujino 2011e] O. Fujino, "Semi-stable minimal model program for varieties with trivial canonical divisor”, Proc. Japan Acad. Ser. A Math. Sci. 87:3 (2011), 25-30. MR 2802603 Zbl 1230.14016

[Fujino and Gongyo 2011] O. Fujino and Y. Gongyo, "Log pluricanonical representations and abundance conjecture”, preprint, 2011. arXiv 1104.0361

[Fujino and Gongyo 2012] O. Fujino and Y. Gongyo, "On canonical bundle formulas and subadjunctions”, Michigan Math. J. 61:2 (2012), 255-264.

[Fukuda 1996] S. Fukuda, "On base point free theorem”, Kodai Math. J. 19:2 (1996), 191-199. MR 97f:14004 Zbl 0860.14005 
[Fukuda 2002] S. Fukuda, "On numerically effective log canonical divisors", Int. J. Math. Math. Sci. 30:9 (2002), 521-531. MR 2003e:14010 Zbl 1058.14027

[Gongyo 2010] Y. Gongyo, "Abundance theorem for numerically trivial log canonical divisors of semi-log canonical pairs", preprint, 2010. To appear in J. Algebraic Geom. arXiv 1005.2796v2

[Iskovskikh 2003] V. A. Iskovskikh, " $b$-divisors and Shokurov functional algebras", Tr. Mat. Inst. Steklova 240 (2003), 8-20. In Russian; translated in Proc. Steklov Inst. Math. 240:1 (2003), 4-15. MR 2004g:14019 Zbl 1081.14022

[Kawamata 1985] Y. Kawamata, "Pluricanonical systems on minimal algebraic varieties", Invent. Math. 79:3 (1985), 567-588. MR 87h:14005 Zbl 0593.14010

[Kawamata 1998] Y. Kawamata, "Subadjunction of log canonical divisors. II", Amer. J. Math. 120:5 (1998), 893-899. MR 2000d:14020 Zbl 0919.14003

[Kawamata 2010] Y. Kawamata, "Variation of mixed Hodge structures and the positivity for algebraic fiber spaces", preprint, 2010. arXiv 1008.1489v1

[Kawamata 2011a] Y. Kawamata, "Hodge theory on generalized normal crossing varieties", preprint, 2011. arXiv 1104.0524v1

[Kawamata 2011b] Y. Kawamata, "Semipositivity theorem for reducible algebraic fiber spaces", Pure Appl. Math. Q. 7:4 (2011), 1427-1447.

[Kawamata et al. 1987] Y. Kawamata, K. Matsuda, and K. Matsuki, "Introduction to the minimal model problem", pp. 283-360 in Algebraic geometry (Sendai, 1985), edited by T. Oda, Adv. Stud. Pure Math. 10, North-Holland, Amsterdam, 1987. MR 89e:14015 Zbl 0672.14006

[Kollár 1993] J. Kollár, "Effective base point freeness", Math. Ann. 296:4 (1993), 595-605. MR 94f: 14004 Zbl 0818.14002

[Kollár 2007] J. Kollár, "Kodaira's canonical bundle formula and adjunction”, pp. 134-162 in Flips for 3-folds and 4-folds, edited by A. Corti, Oxford Lecture Ser. Math. Appl. 35, Oxford Univ. Press, 2007. MR 2359346

[Lazarsfeld 2004] R. Lazarsfeld, Positivity in algebraic geometry, II: Positivity for vector bundles, and multiplier ideals, Ergebnisse der Math. (3) 49, Springer, Berlin, 2004. MR 2005k:14001b Zbl 1093.14500

[Nakayama 1986] N. Nakayama, "Invariance of the plurigenera of algebraic varieties under minimal model conjectures", Topology 25:2 (1986), 237-251. MR 87g:14034 Zbl 0596.14026

[Shokurov 2003] V. V. Shokurov, "Prelimiting flips", Tr. Mat. Inst. Steklova 240 (2003), 82-219. Reprinted in Proc. Steklov Inst. Math. 240: (2003), 75-213. MR 2004k:14024 Zbl 1082.14019

Communicated by Shigefumi Mori

Received 2011-04-30 Revised 2011-05-18 Accepted 2011-06-20

fujino@math.kyoto-u.ac.jp Department of Mathematics, Faculty of Science, Kyoto University, Sakyo-ku Kitashirakawa, Oiwake-cho, Kyoto 606-8502, Japan 


\section{Algebra \& Number Theory}

msp.berkeley.edu/ant

\section{EDITORS}

MANAGING EDITOR

Bjorn Poonen

Massachusetts Institute of Technology

Cambridge, USA

\author{
EDITORIAL BOARD CHAIR \\ David Eisenbud \\ University of California \\ Berkeley, USA
}

\section{BOARD OF EDITORS}

Georgia Benkart

Dave Benson

Richard E. Borcherds

John H. Coates

J-L. Colliot-Thélène

Brian D. Conrad

Hélène Esnault

Hubert Flenner

Edward Frenkel

Andrew Granville

Joseph Gubeladze

Ehud Hrushovski

Craig Huneke

Mikhail Kapranov

Yujiro Kawamata

János Kollár

Yuri Manin

Barry Mazur

Philippe Michel

Susan Montgomery
University of Wisconsin, Madison, USA

University of Aberdeen, Scotland

University of California, Berkeley, USA

University of Cambridge, UK

CNRS, Université Paris-Sud, France

University of Michigan, USA

Universität Duisburg-Essen, Germany

Ruhr-Universität, Germany

University of California, Berkeley, USA

Université de Montréal, Canada

San Francisco State University, USA

Hebrew University, Israel

University of Kansas, USA

Yale University, USA

University of Tokyo, Japan

Princeton University, USA

Northwestern University, USA

Harvard University, USA

École Polytechnique Fédérale de Lausanne

University of Southern California, USA
Shigefumi Mori

Raman Parimala

Jonathan Pila

Victor Reiner

Karl Rubin

Peter Sarnak

Joseph H. Silverman

Michael Singer

Ronald Solomon

Vasudevan Srinivas

J. Toby Stafford

Bernd Sturmfels

Richard Taylor

Ravi Vakil

Michel van den Bergh

Marie-France Vignéras

Kei-Ichi Watanabe

Andrei Zelevinsky

Efim Zelmanov
RIMS, Kyoto University, Japan

Emory University, USA

University of Oxford, UK

University of Minnesota, USA

University of California, Irvine, USA

Princeton University, USA

Brown University, USA

North Carolina State University, USA

Ohio State University, USA

Tata Inst. of Fund. Research, India

University of Michigan, USA

University of California, Berkeley, USA

Harvard University, USA

Stanford University, USA

Hasselt University, Belgium

Université Paris VII, France

Nihon University, Japan

Northeastern University, USA

University of California, San Diego, USA

\section{PRODUCTION}

contact@msp.org

Silvio Levy, Scientific Editor

See inside back cover or www.jant.org for submission instructions.

The subscription price for 2012 is US \$175/year for the electronic version, and \$275/year (+\$40 shipping outside the US) for print and electronic. Subscriptions, requests for back issues from the last three years and changes of subscribers address should be sent to Mathematical Sciences Publishers, Department of Mathematics, University of California, Berkeley, CA 94720-3840, USA.

Algebra \& Number Theory (ISSN 1937-0652) at Mathematical Sciences Publishers, Department of Mathematics, University of California, Berkeley, CA 94720-3840 is published continuously online. Periodical rate postage paid at Berkeley, CA 94704, and additional mailing offices.

ANT peer review and production are managed by EditFLOW ${ }^{\circledR}$ from Mathematical Sciences Publishers.

PUBLISHED BY

mathematical sciences publishers

http://msp.org/

A NON-PROFIT CORPORATION

Typeset in IAT $_{\mathrm{E}} \mathrm{X}$

Copyright (C2012 by Mathematical Sciences Publishers 


\section{Algebra \& Number Theory}

Volume $6 \quad$ No. $4 \quad 2012$

Spherical varieties and integral representations of $L$-functions

YIANNIS SAKELLARIDIS

Nonuniruledness results for spaces of rational curves in hypersurfaces

ROYA BEHESHTI

Degeneracy of triality-symmetric morphisms

DAVE ANDERSON

Multi-Frey $\mathbb{Q}$-curves and the Diophantine equation $a^{2}+b^{6}=c^{n}$

MiChaEl A. BENNETT and IMIN CHEN

Detaching embedded points

DAWEI CHEN and SCOTT NOLLET

Moduli of Galois $p$-covers in mixed characteristics

DAN ABRAMOVICH and MATTHIEU ROMAGNY

Block components of the Lie module for the symmetric group

ROGER M. BRYANT and KARIN ERDMANN

Basepoint-free theorems: saturation, b-divisors, and canonical bundle formula

OSAMU FUJINO

Realizing large gaps in cohomology for symmetric group modules

DAVID J. HEMMER 